Supporting information for

\title{
Parallel Multistream Training of High-Dimensional Neural Network Potentials
}

\author{
Andreas Singraber ${ }^{\mathrm{a}}$, Tobias Morawietz ${ }^{\mathrm{b}}$, Jörg Behler ${ }^{\mathrm{c}}$, Christoph Dellago $^{\mathrm{a}}$ \\ ${ }^{a}$ Faculty of Physics, University of Vienna, Boltzmanngasse 5, Vienna, Austria \\ ${ }^{\mathrm{b}}$ Department of Chemistry, Stanford University, Stanford, California 94305, United States \\ ${ }^{\mathrm{c}}$ Universität Göttingen, Institut für Physikalische Chemie, Theoretische Chemie, \\ Tammannstraße 6, 37077 Göttingen, Germany
}

\section{Contents}

\begin{tabular}{lll}
\hline 1 & Kalman filter parameter study results & 2
\end{tabular}

\begin{tabular}{lll}
\hline 2 & Training time comparison & 15
\end{tabular}

\begin{tabular}{|lll}
3 & $\mathrm{Cu}_{2} \mathrm{~S}$ HDNNP symmetry function parameters & 16
\end{tabular}

\begin{tabular}{|lll}
4 & Low chalcocite structure comparison & 20
\end{tabular}

\begin{tabular}{|lll}
5 & Copper diffusion coefficient values & 22
\end{tabular}

\begin{tabular}{ll}
\hline References & 23
\end{tabular} 


\section{Kalman filter parameter study results}

In this section we present additional results from the Kalman filter parameter study discussed in the main text. There, only the median RMSEs of energies and forces for training runs with 16 parallel streams using the global weight update scheme were illustrated (corresponding to Figures $3 \mathrm{c}$ and $6 \mathrm{c}$ but with different color scale limits). Here, we add a large number of similar plots combining all of the following settings:

- Weight update scheme:

- Global (Figures 1 to 6)

- Per-element (Figures 7 to 12 )

- Physical quantity:

- Energies (Figures 1, 2, 3, 7, 8, 9)

- Forces (Figures 4, 5, 6, 10, 11, 12)

- RMSE criterion:

- Best of all training runs (Figures 1, 4, 7, 10)

- Worst of all training runs (Figures 2, 5, 8, 11)

- Median of all training runs (Figures 3, 6, 9, 12)

- Number of streams:

- 1, identical to regular Kalman filter (subfigure (a) on each page)

- 4 (subfigure (b) on each page)

- 16 (subfigure (c) on each page)

- 32 (subfigure (d) on each page)

- 48 (subfigure (e) on each page)

- 64 (subfigure (f) on each page)

The subfigures (a) - (f) on each page share the same color bar limits to make comparison between different stream selections easier. Furthermore, to visualize the superior performance of the global weight update scheme over the per-element version, we use identical color scales for matching figure pairs, e.g. Figures 1 and 7 , 2 and 8 , and so on. 

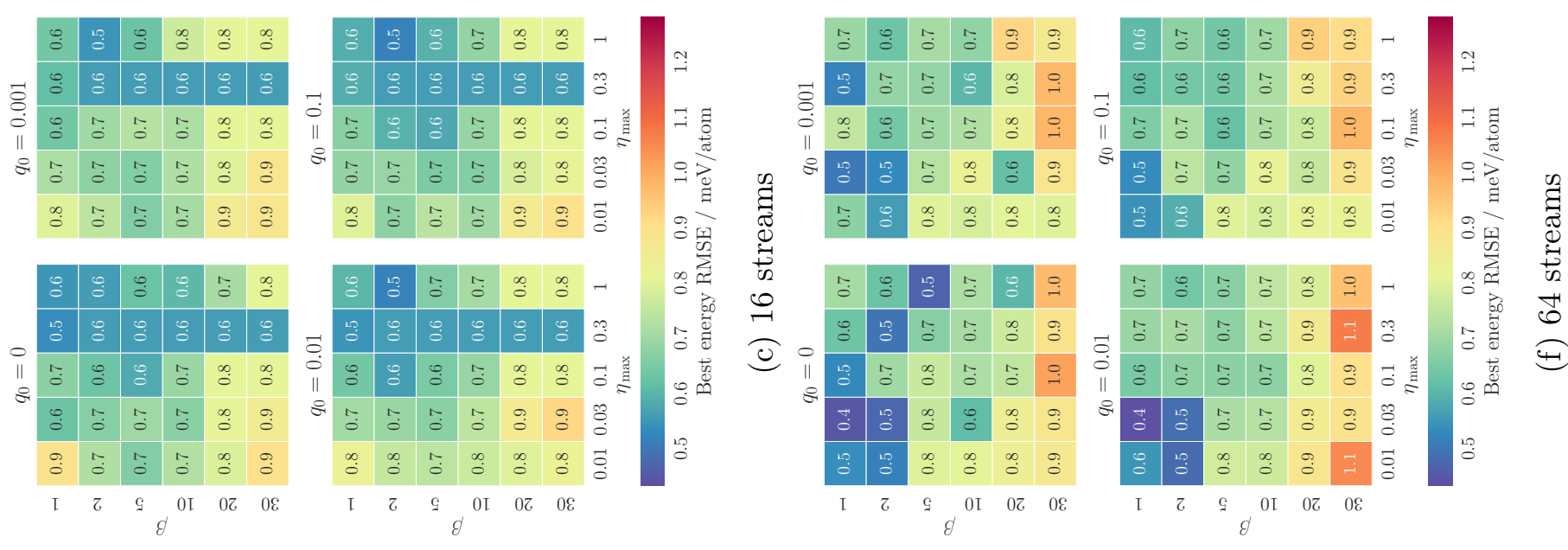

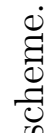
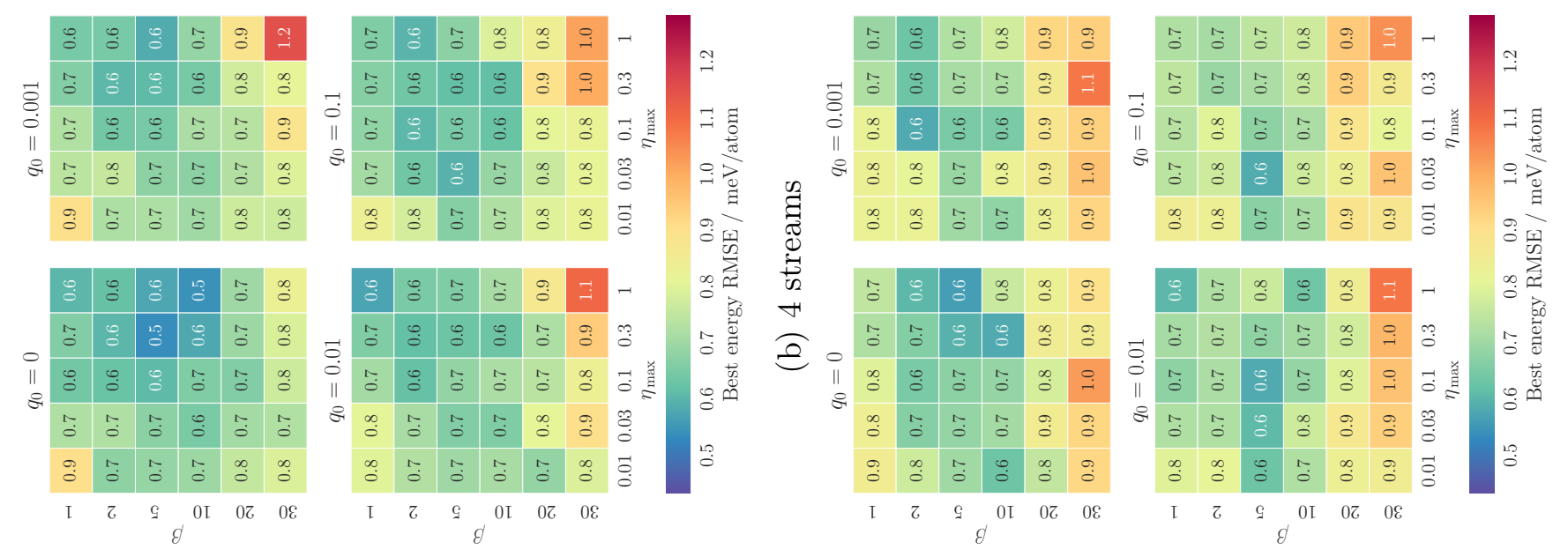

s,

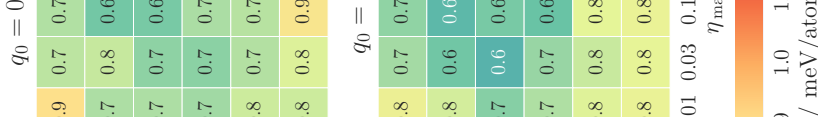
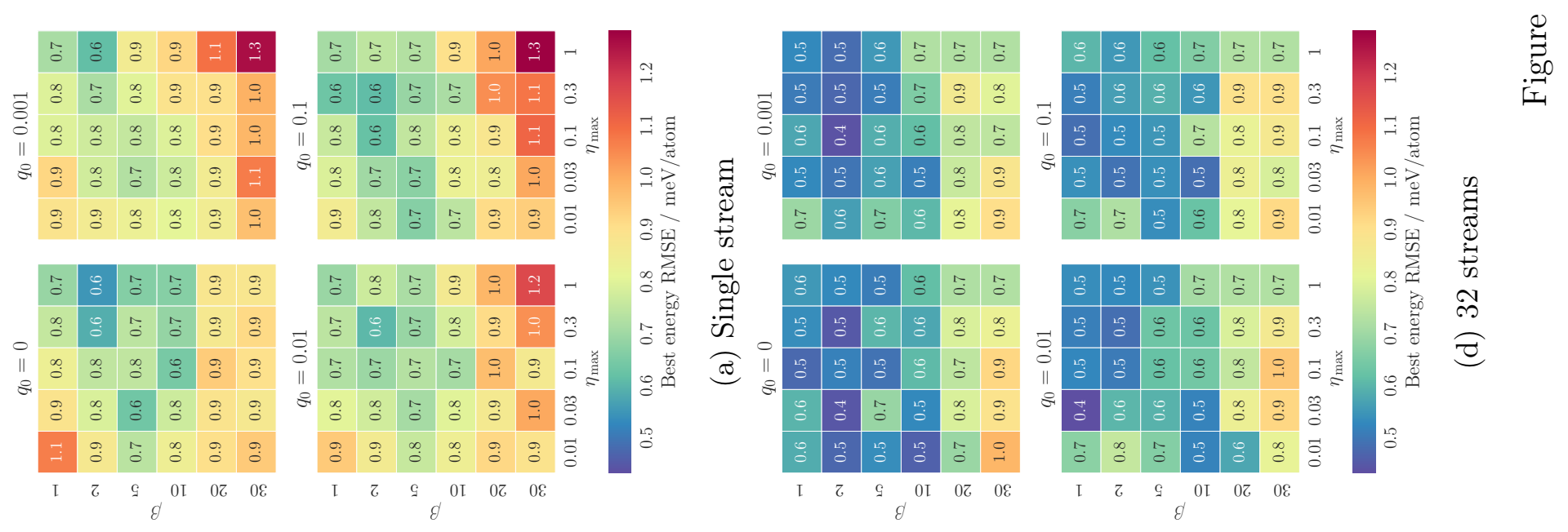

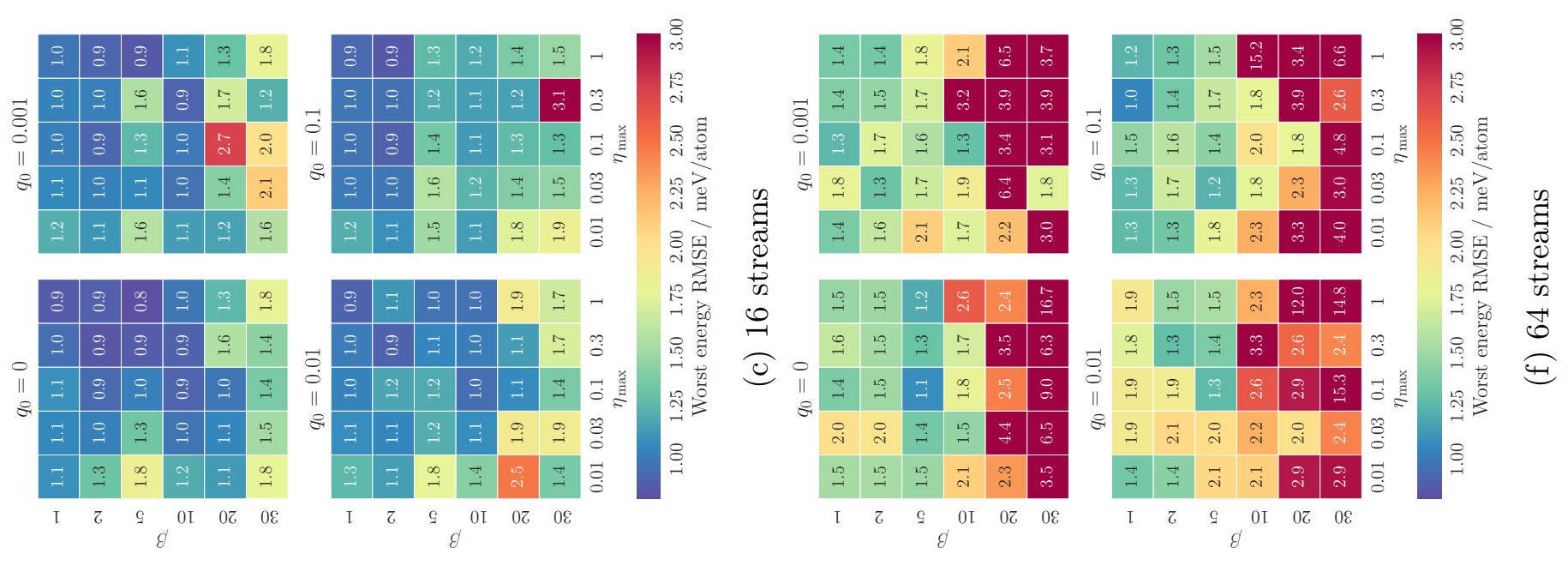

总
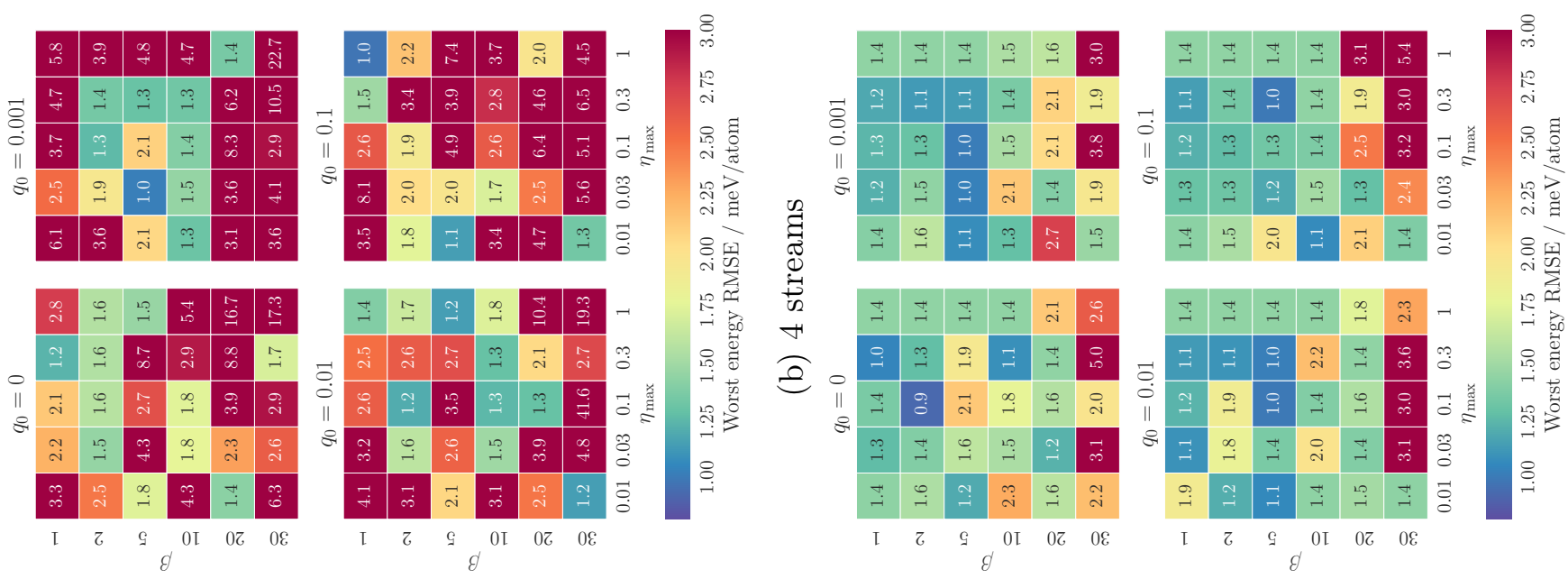

䓌
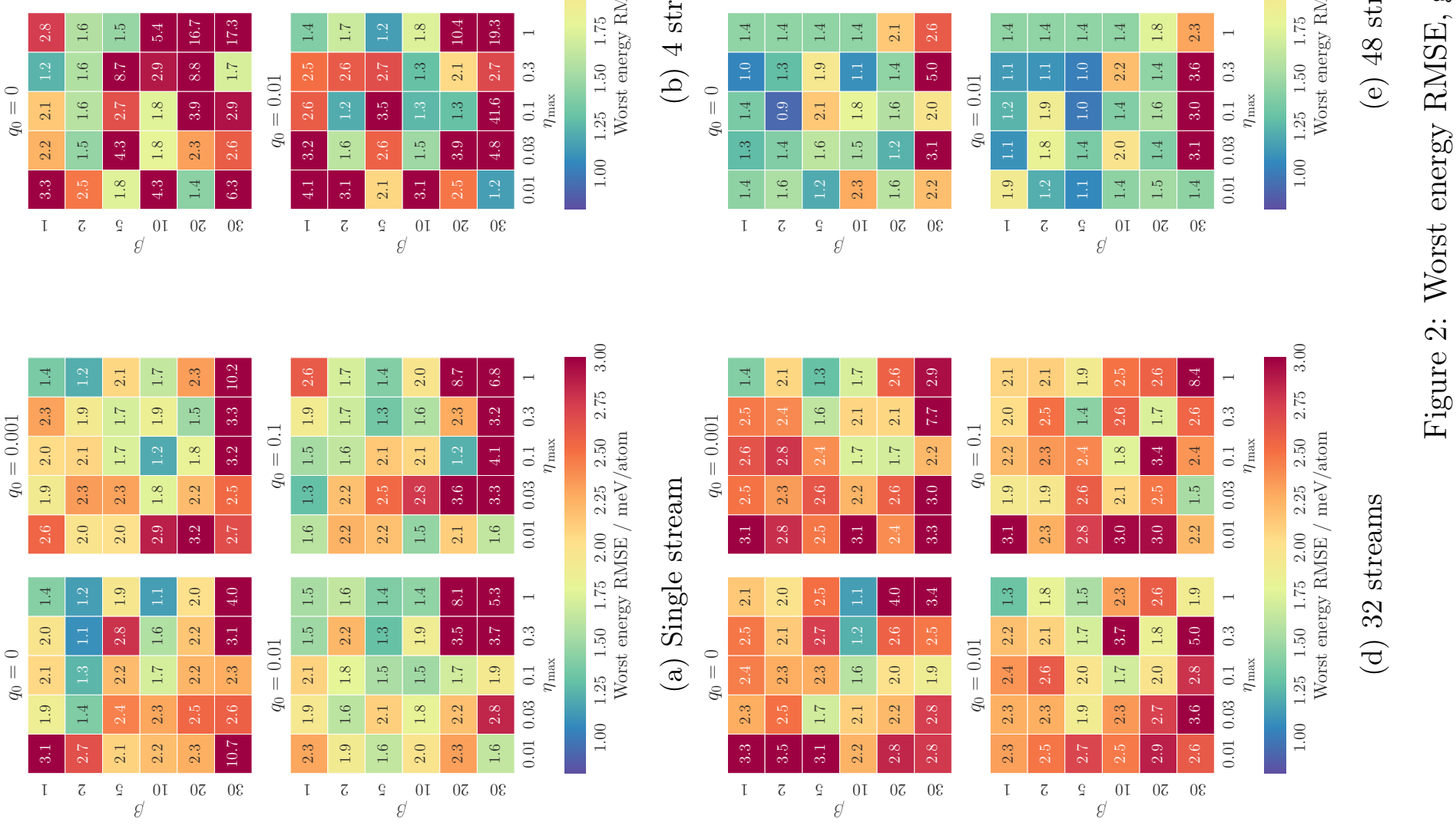

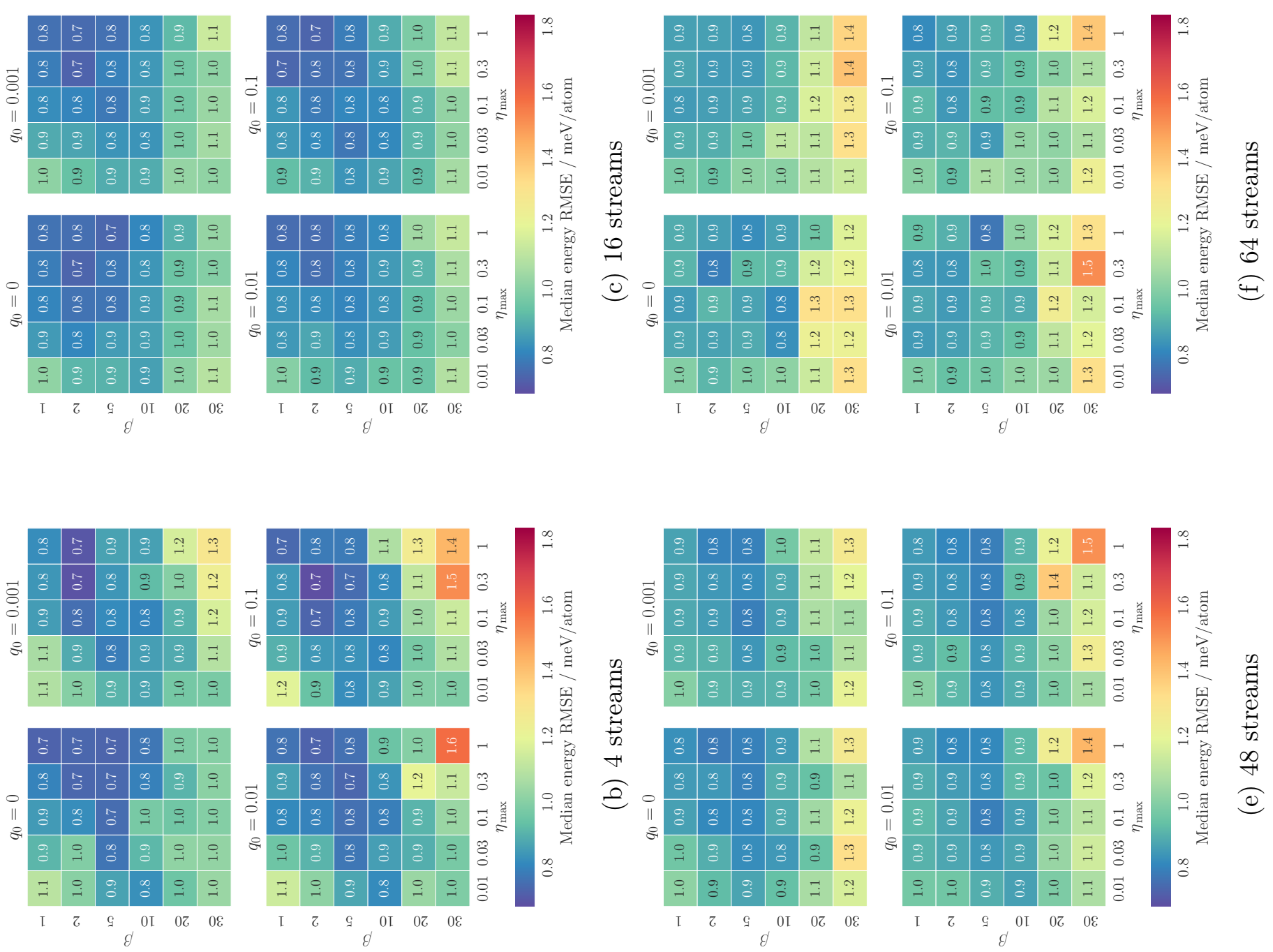

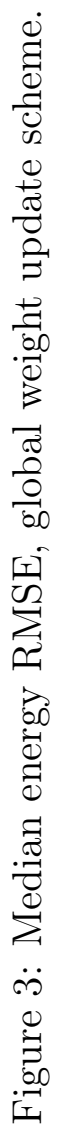
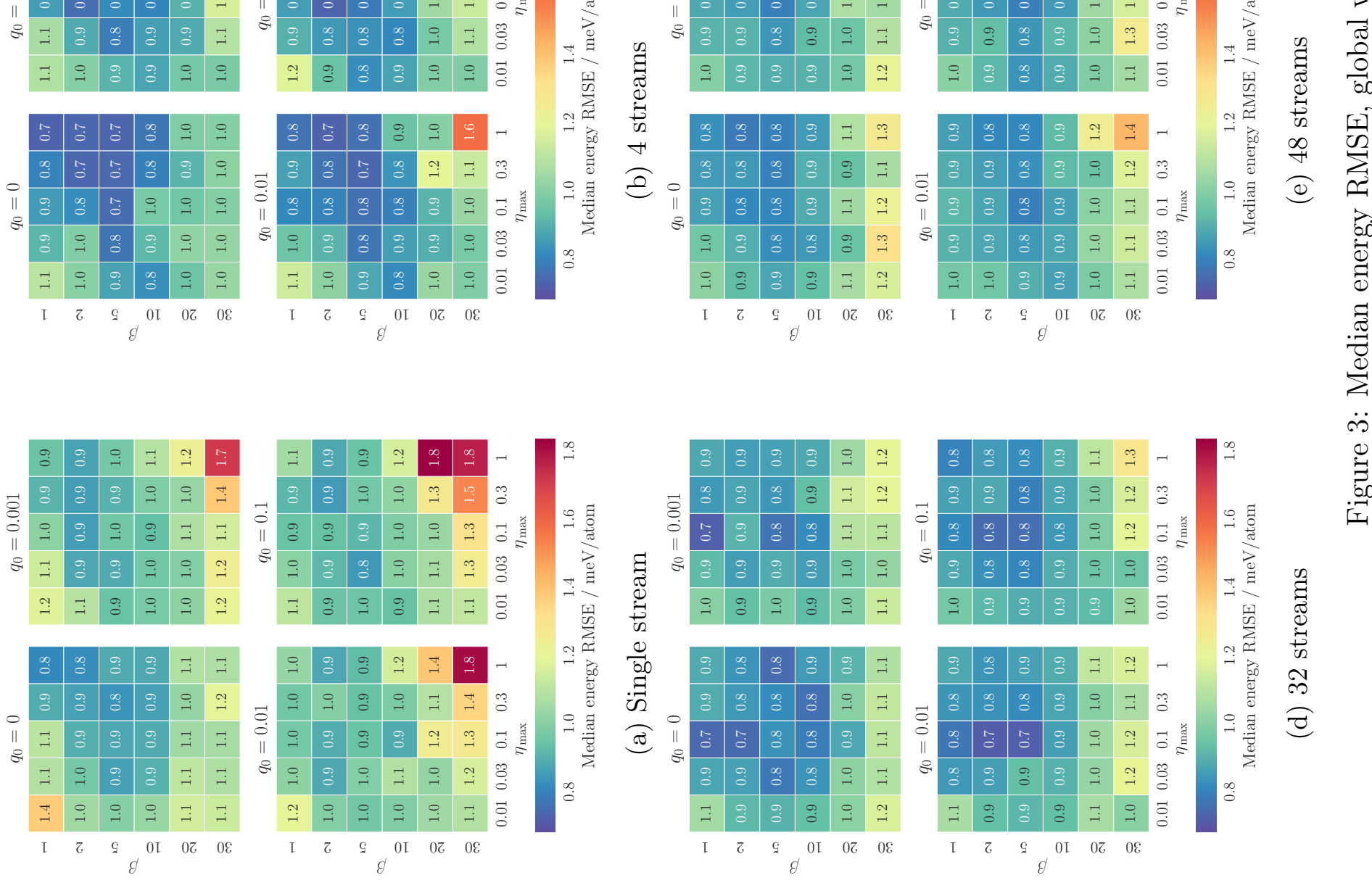

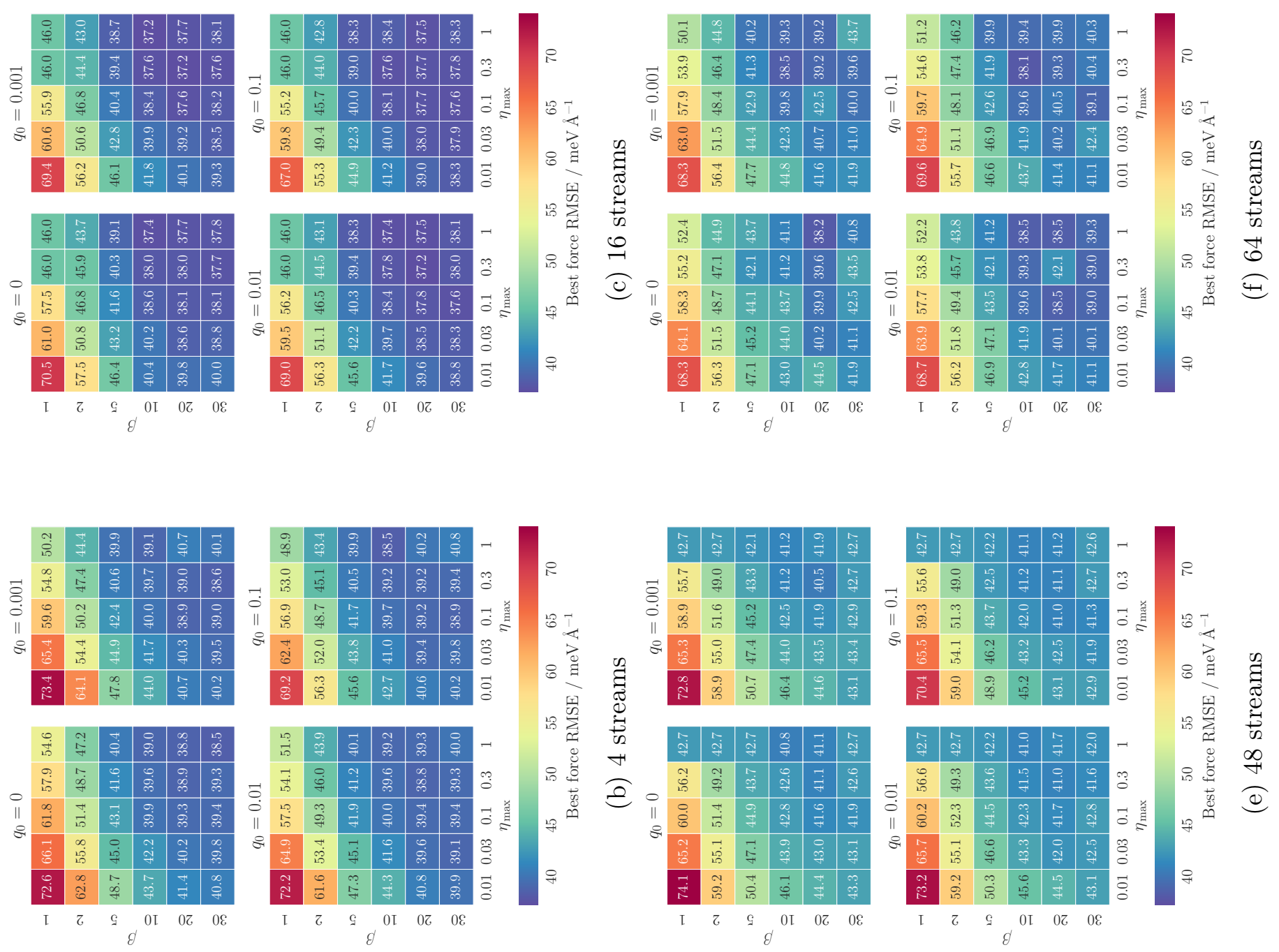

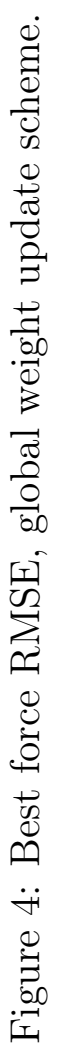
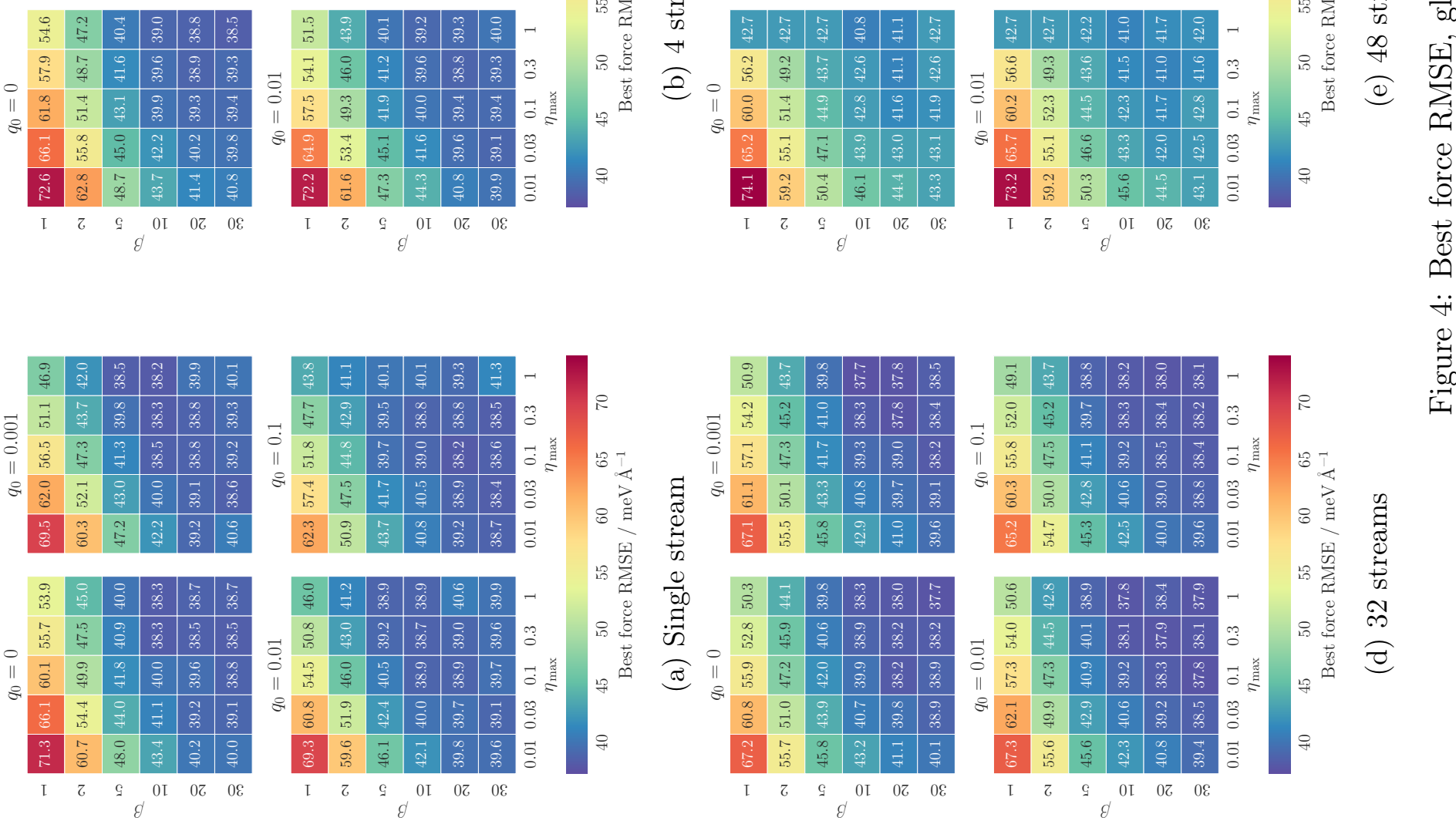

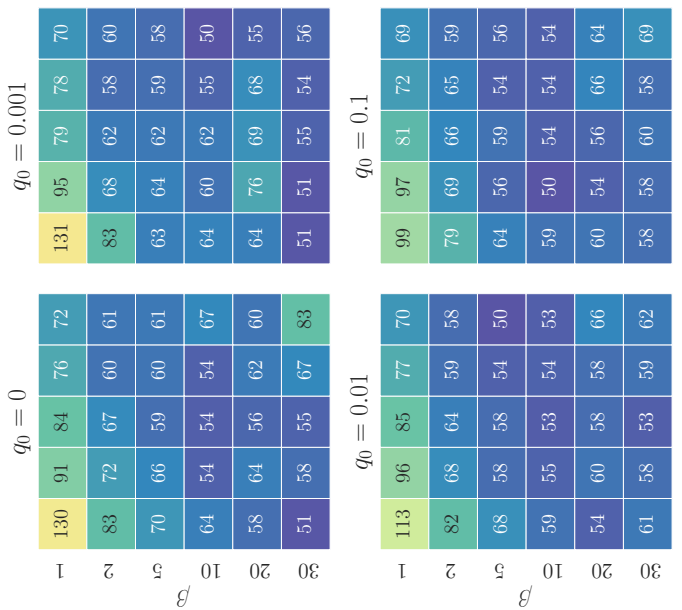

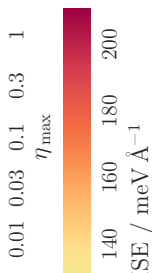

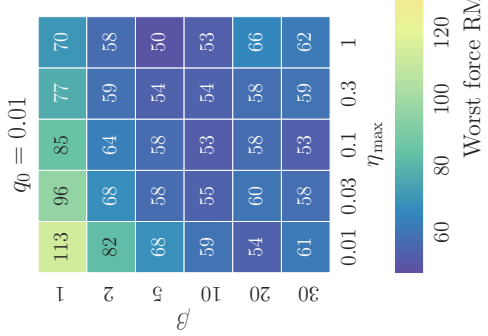

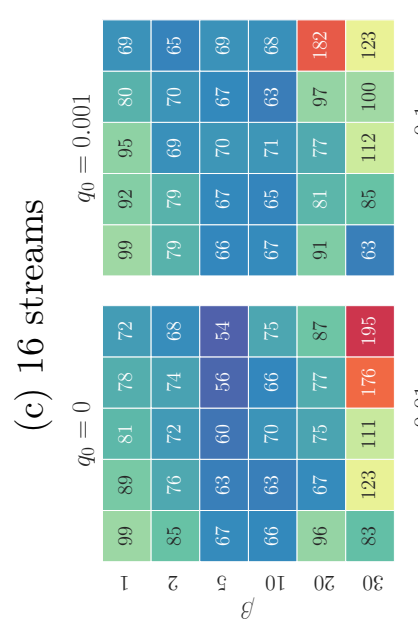
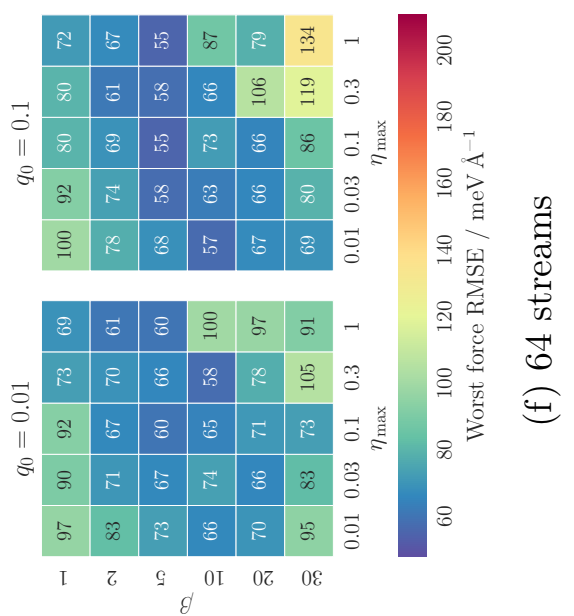

过
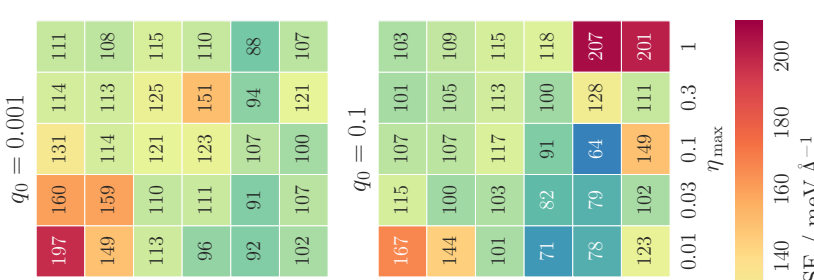

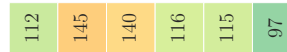
○占哭梁苏点

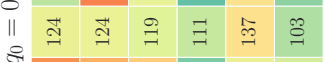

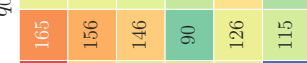

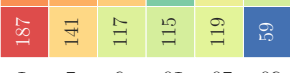

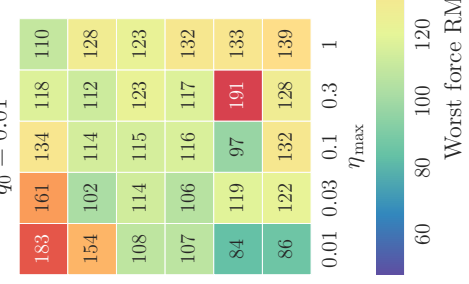
0
8
8
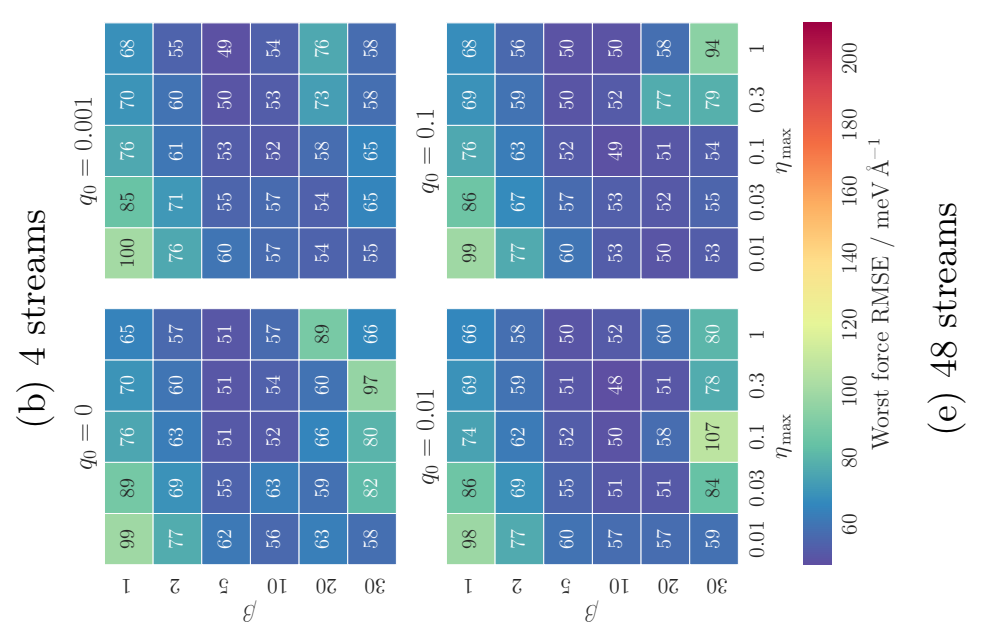

宽
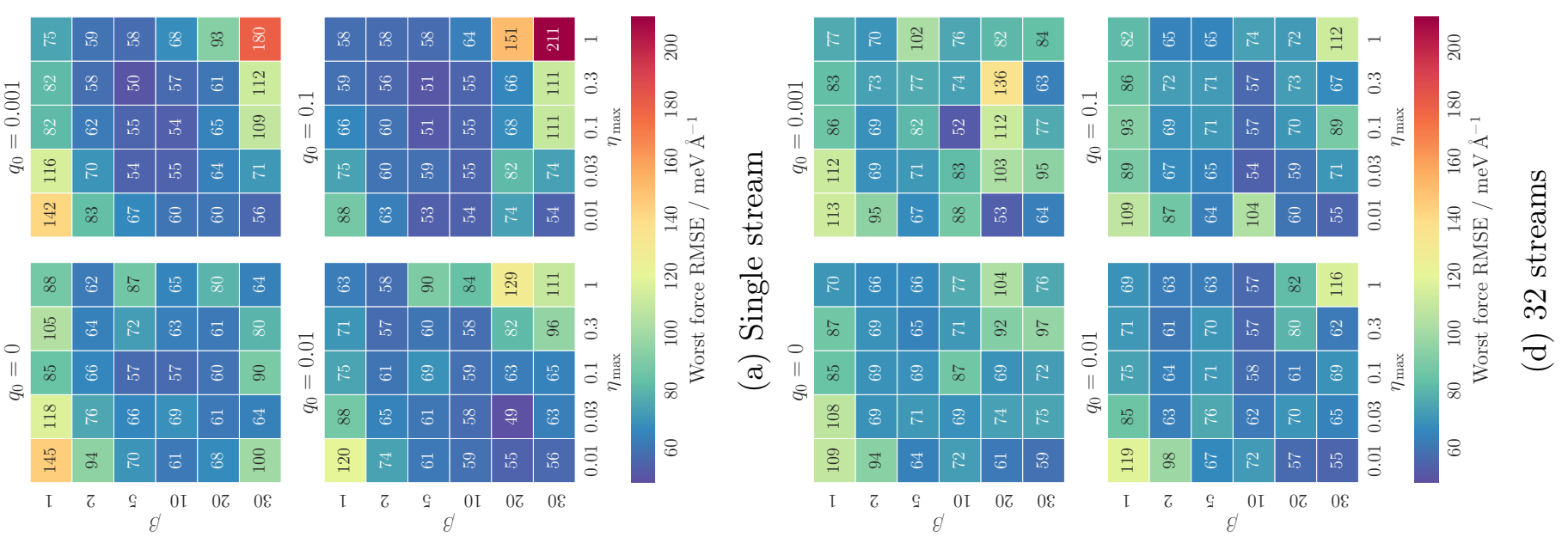

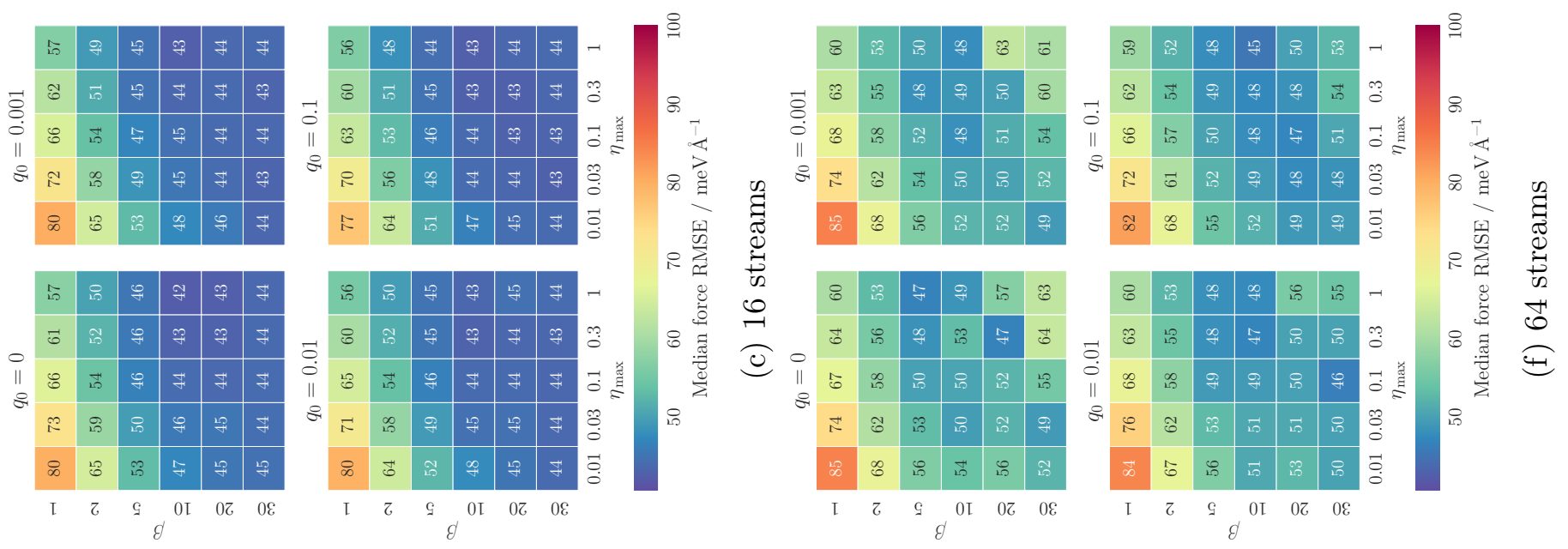

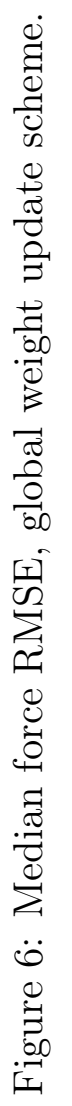
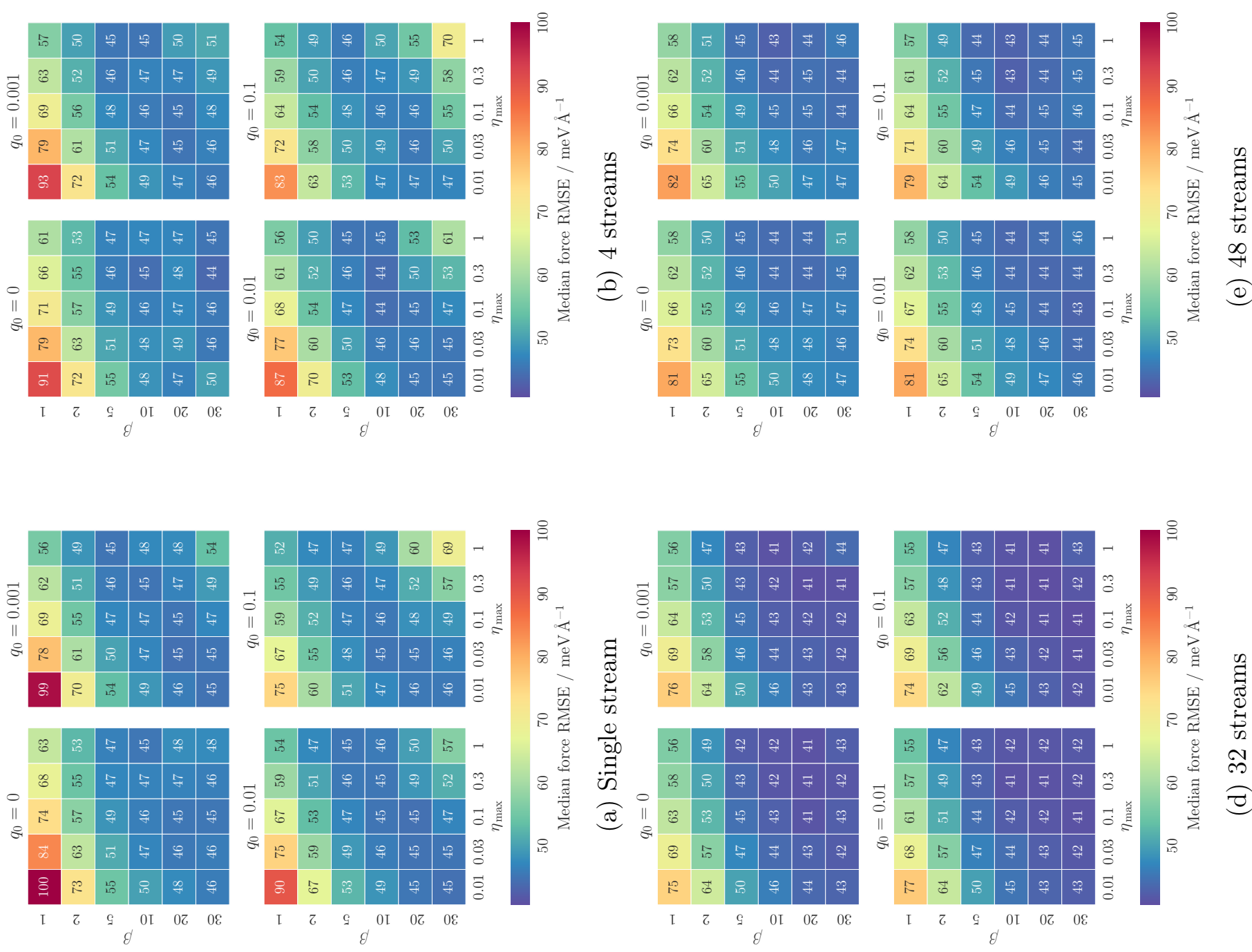

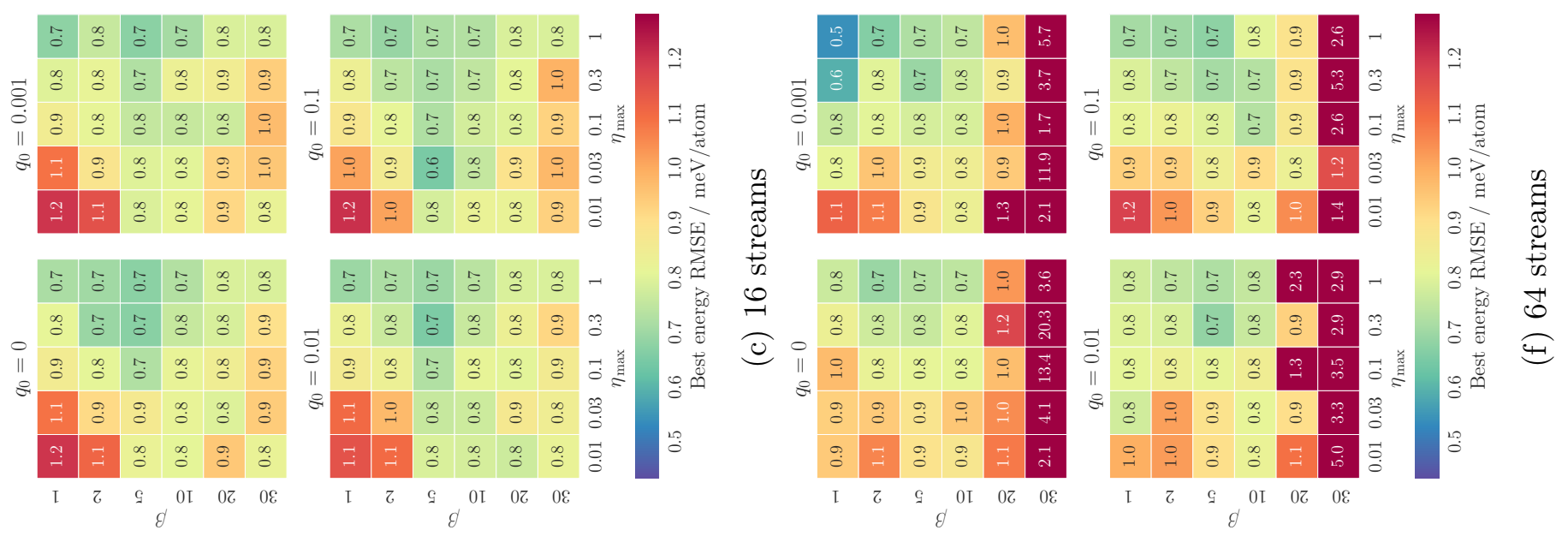

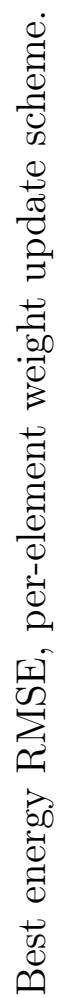

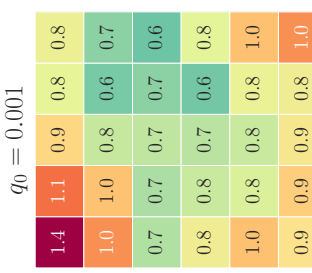
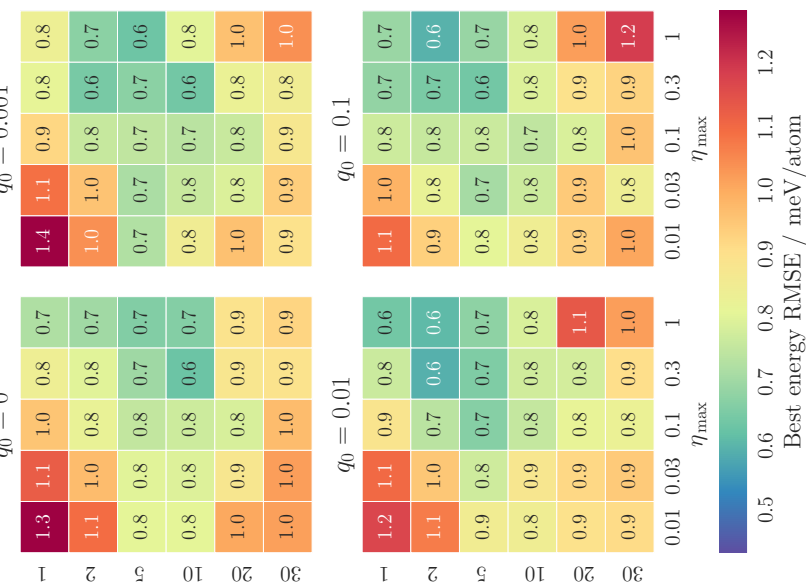
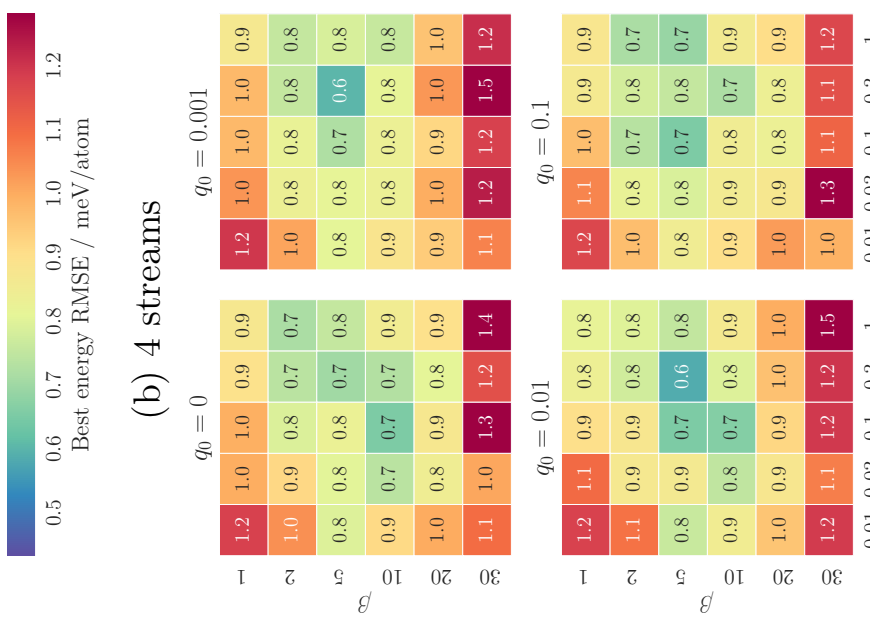
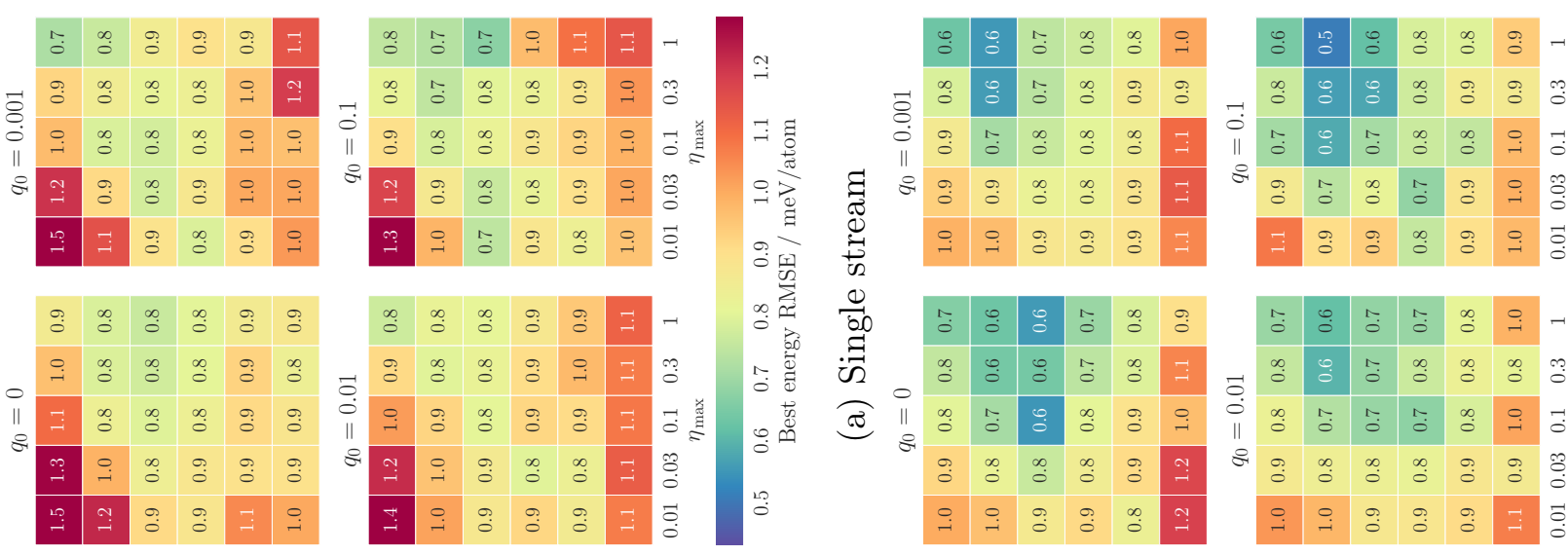

in
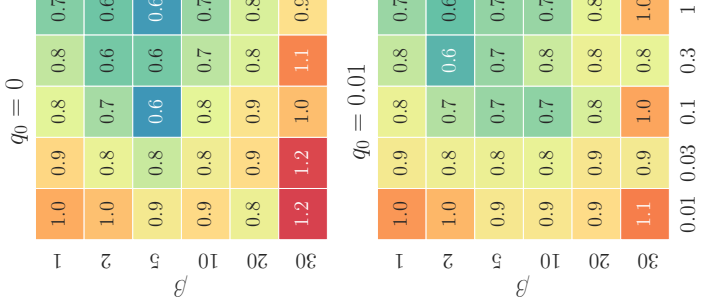

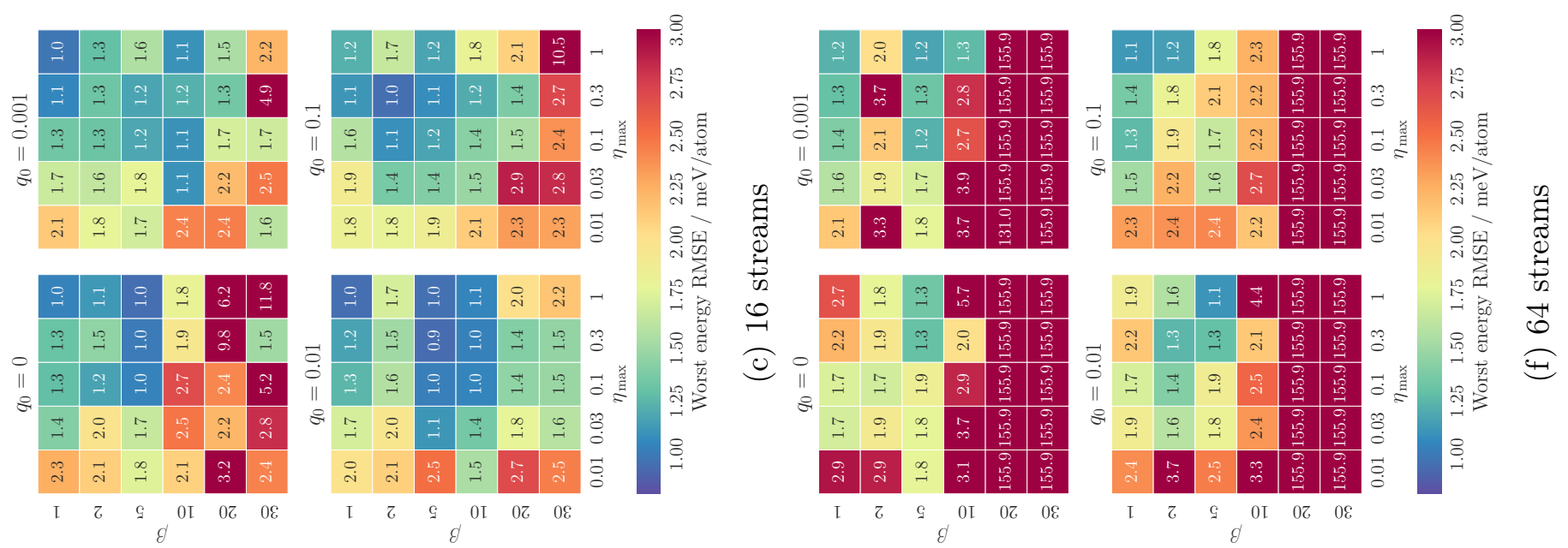

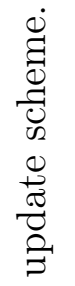
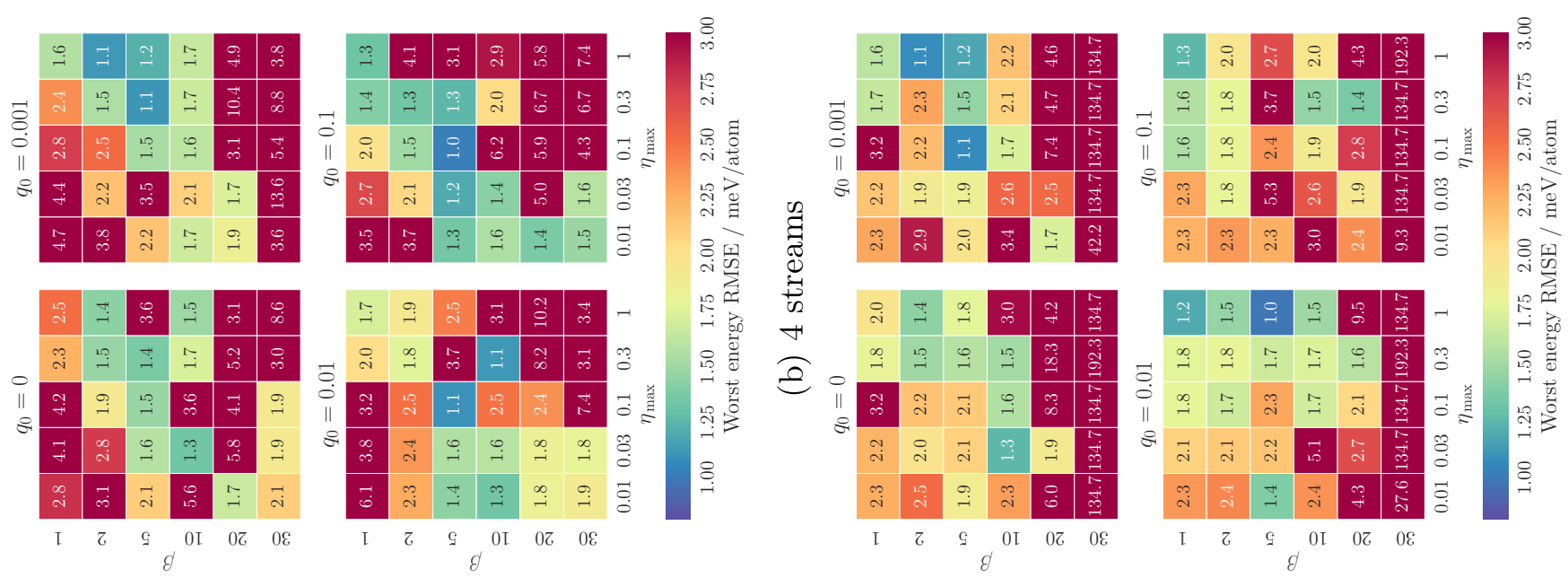

$\frac{7}{20}$
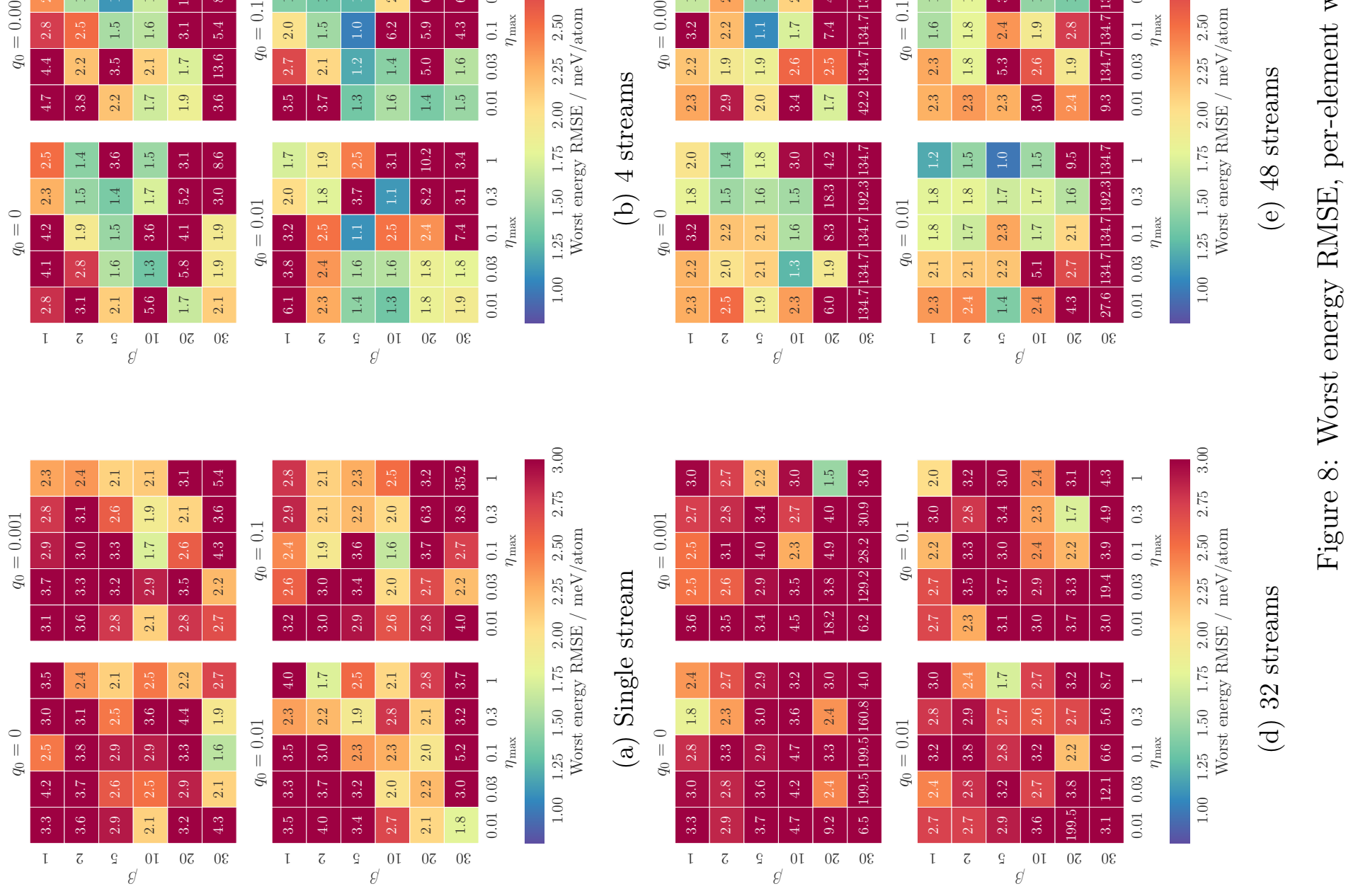

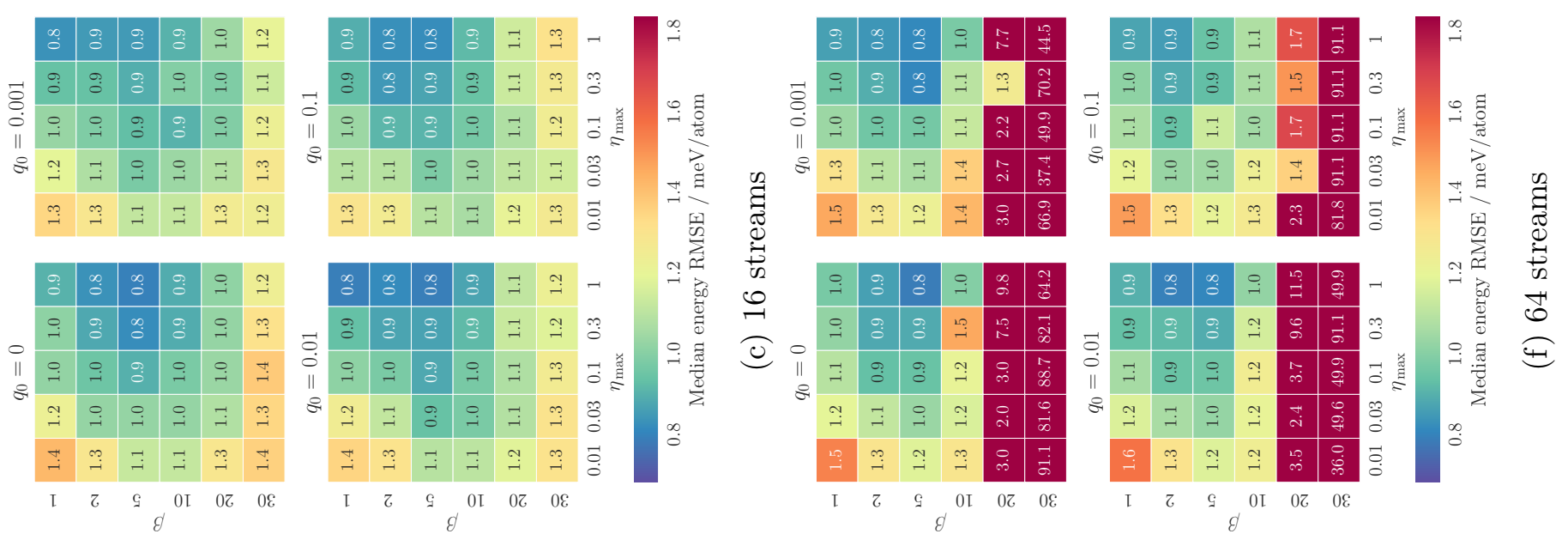

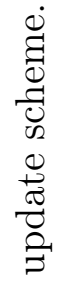
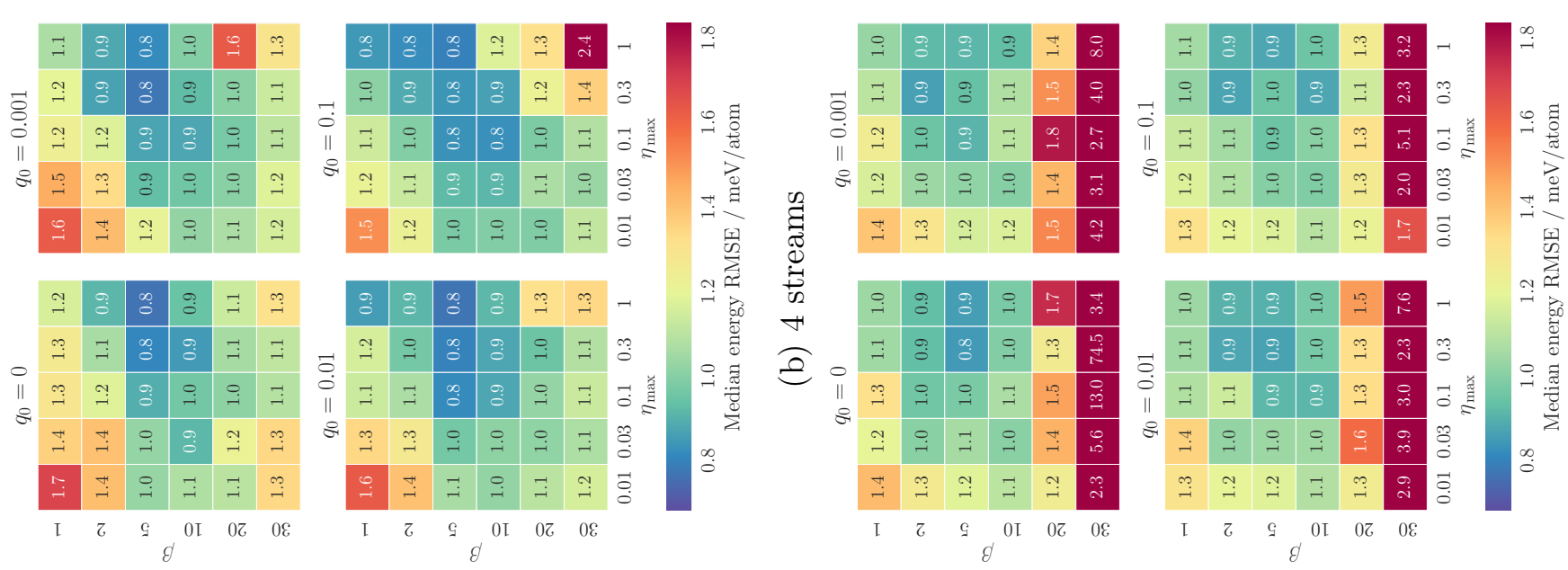

4
.00
00
0
3
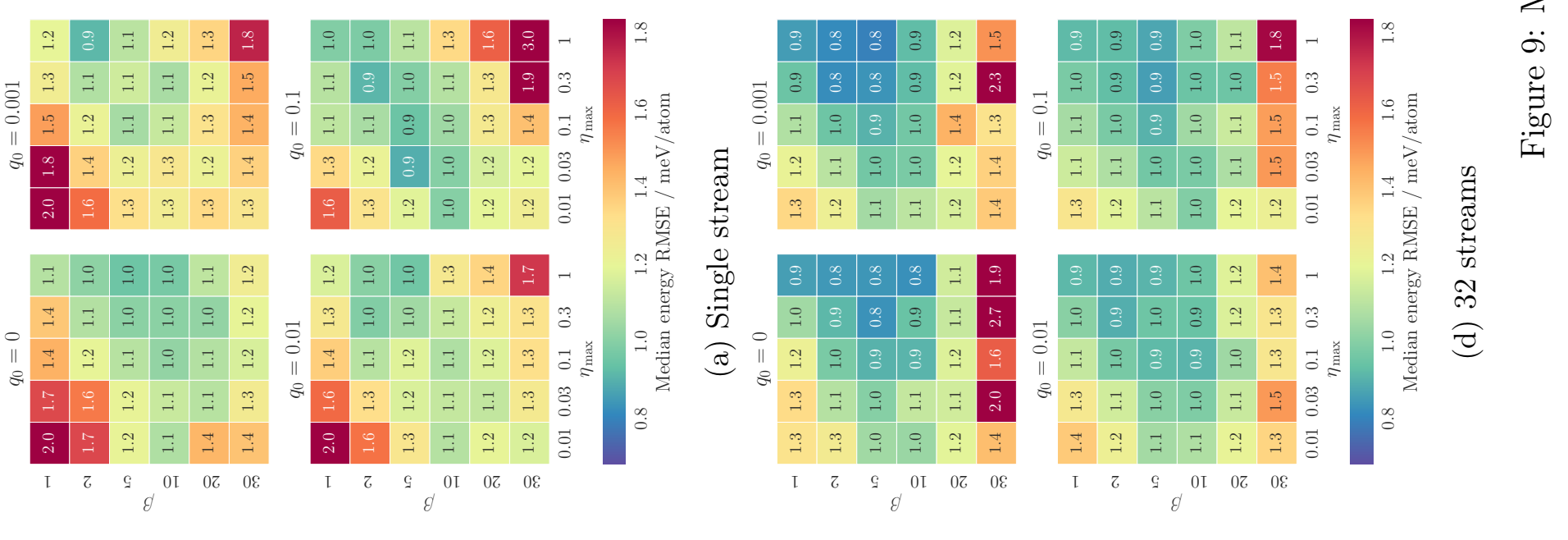

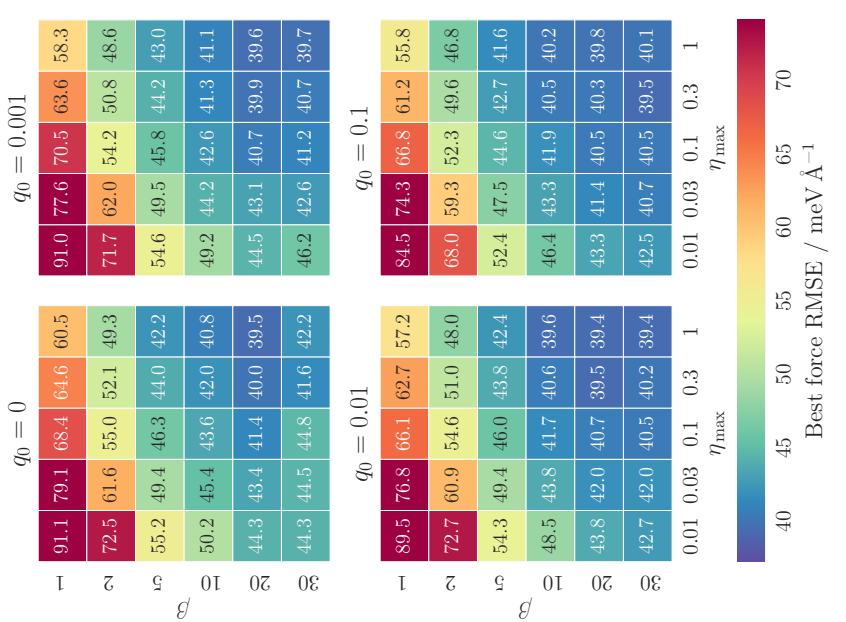
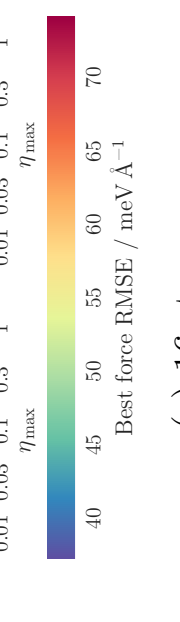
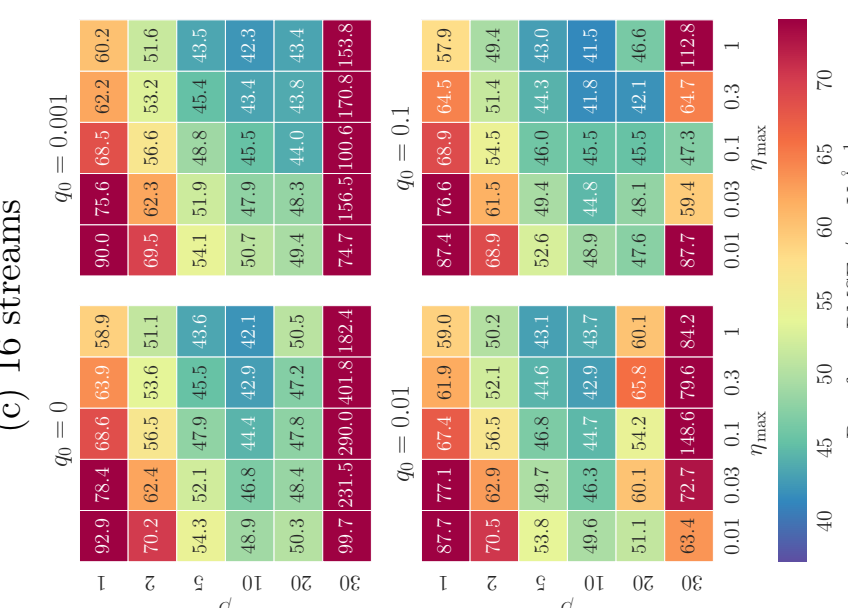
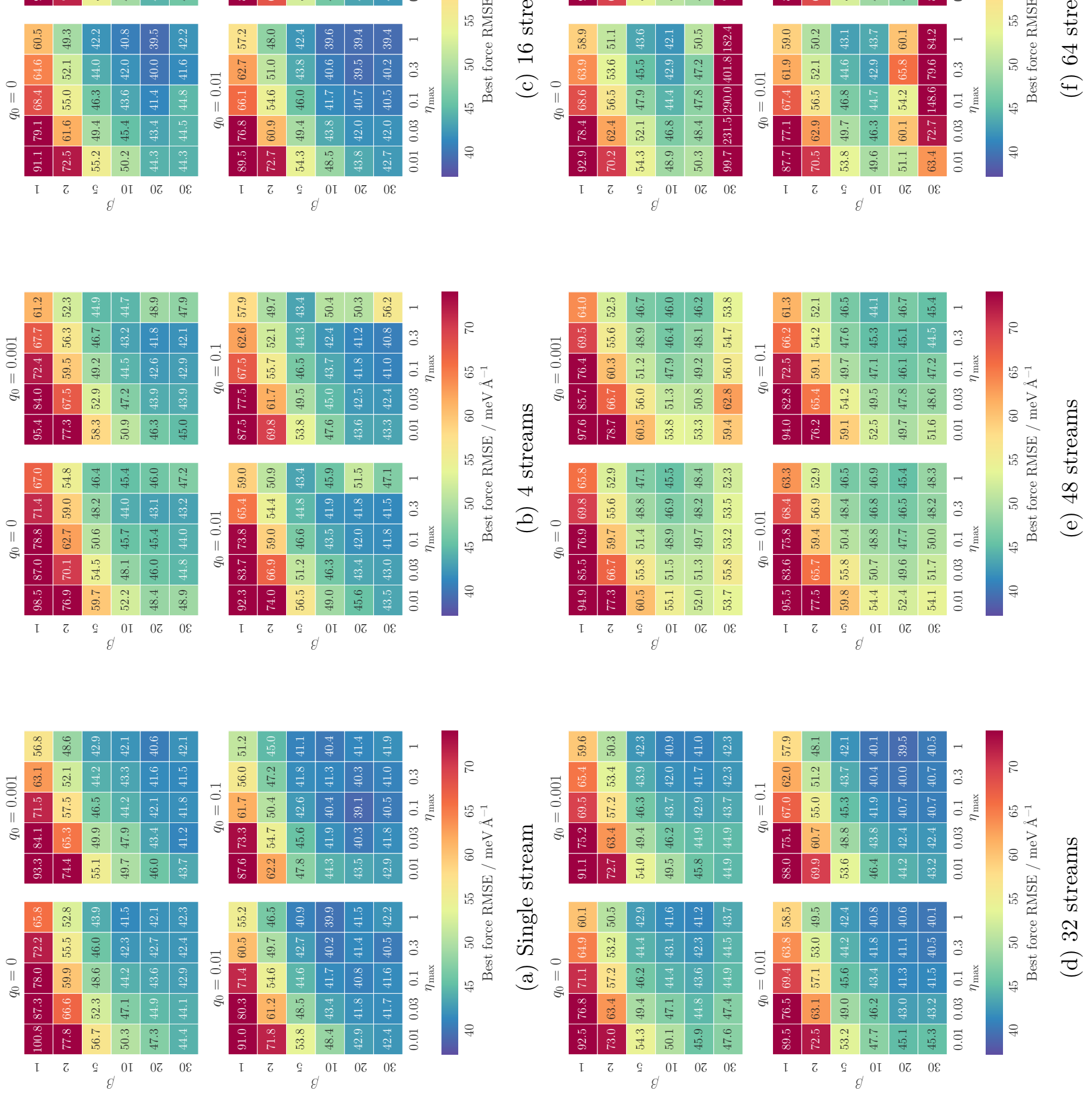
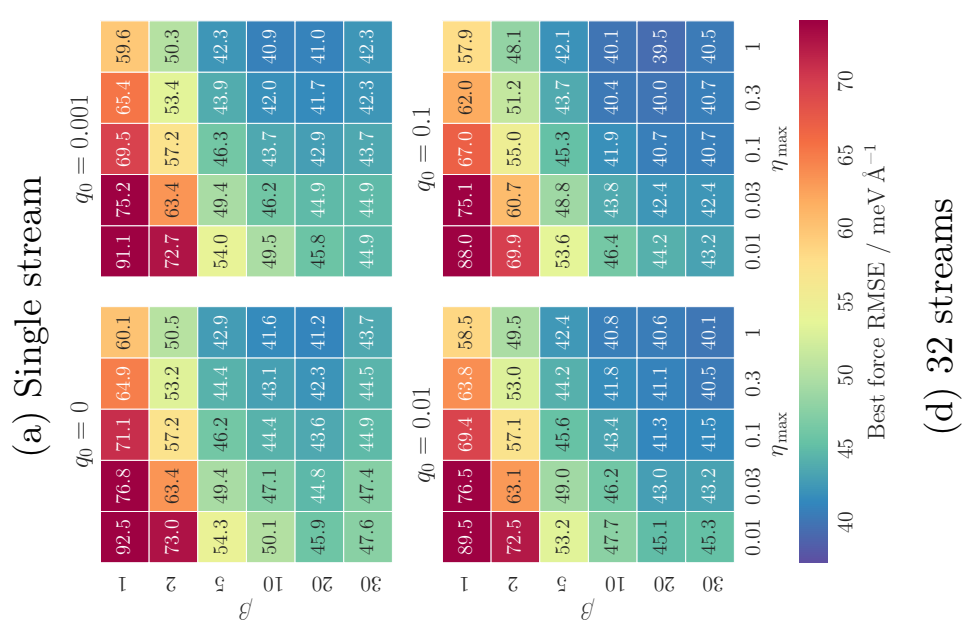
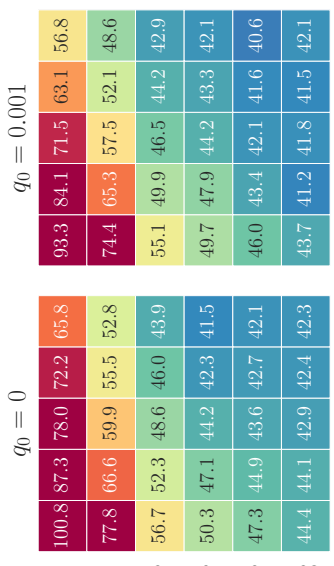

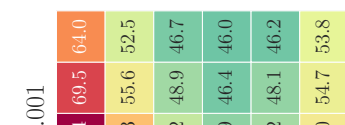
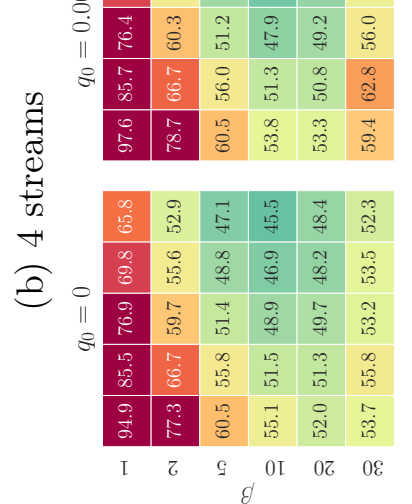

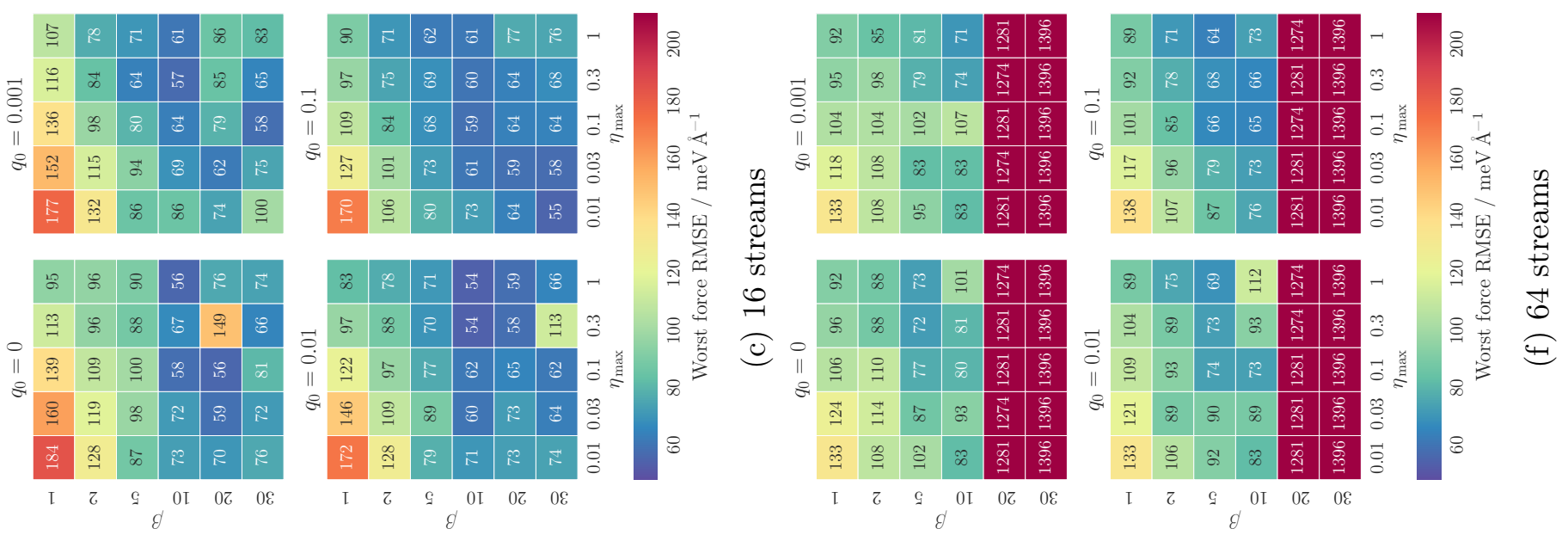

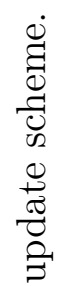
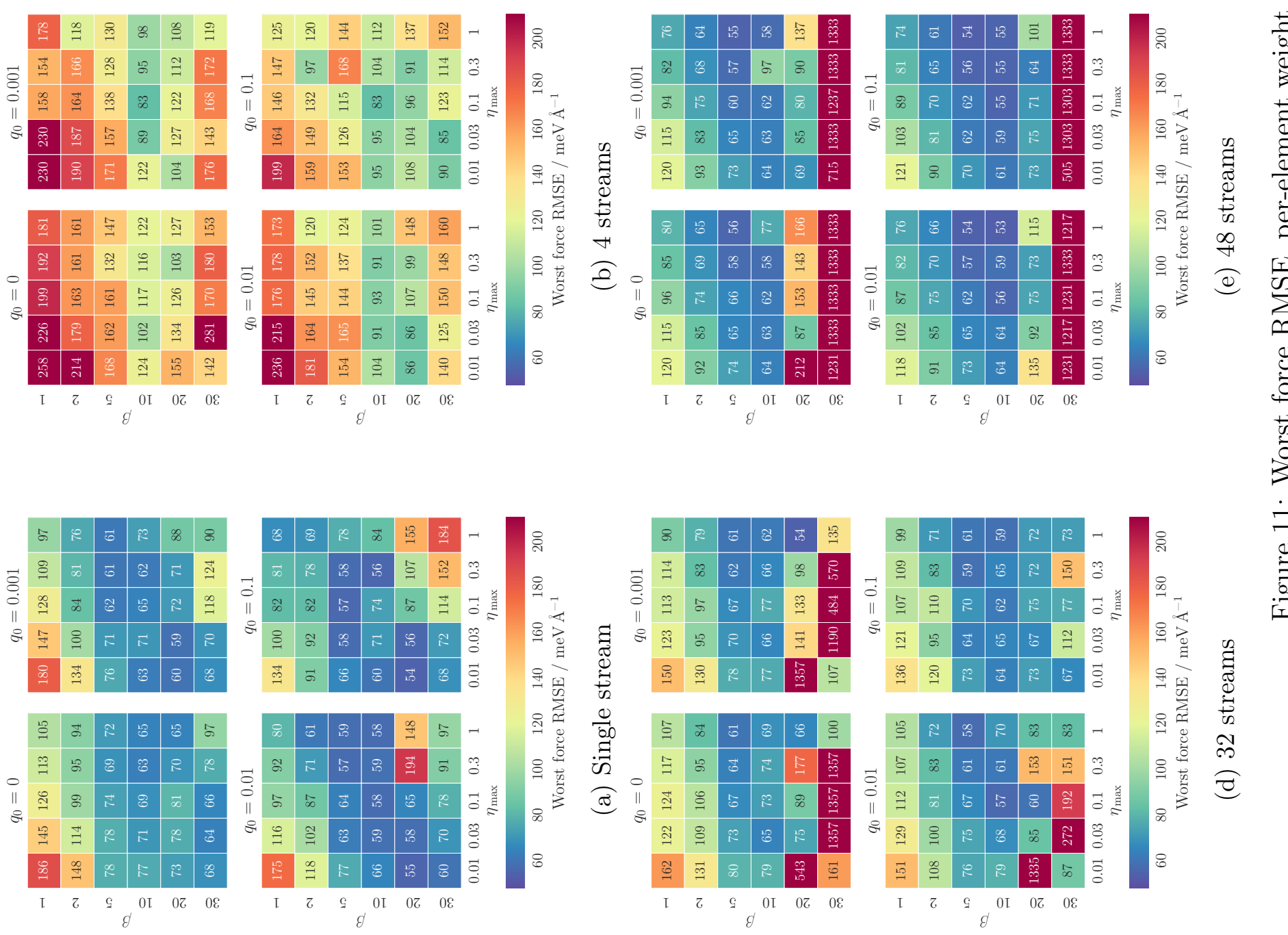

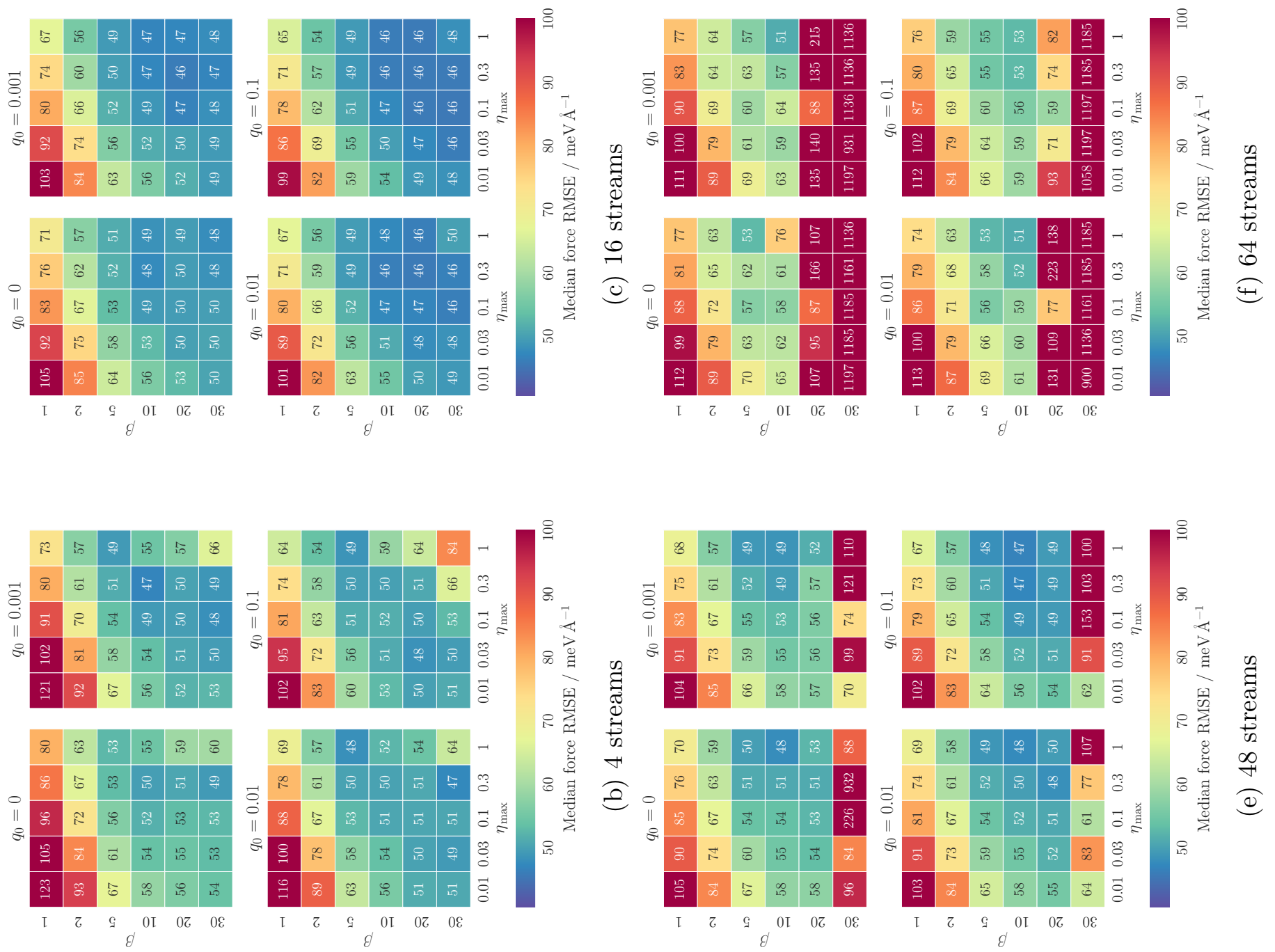

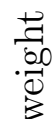
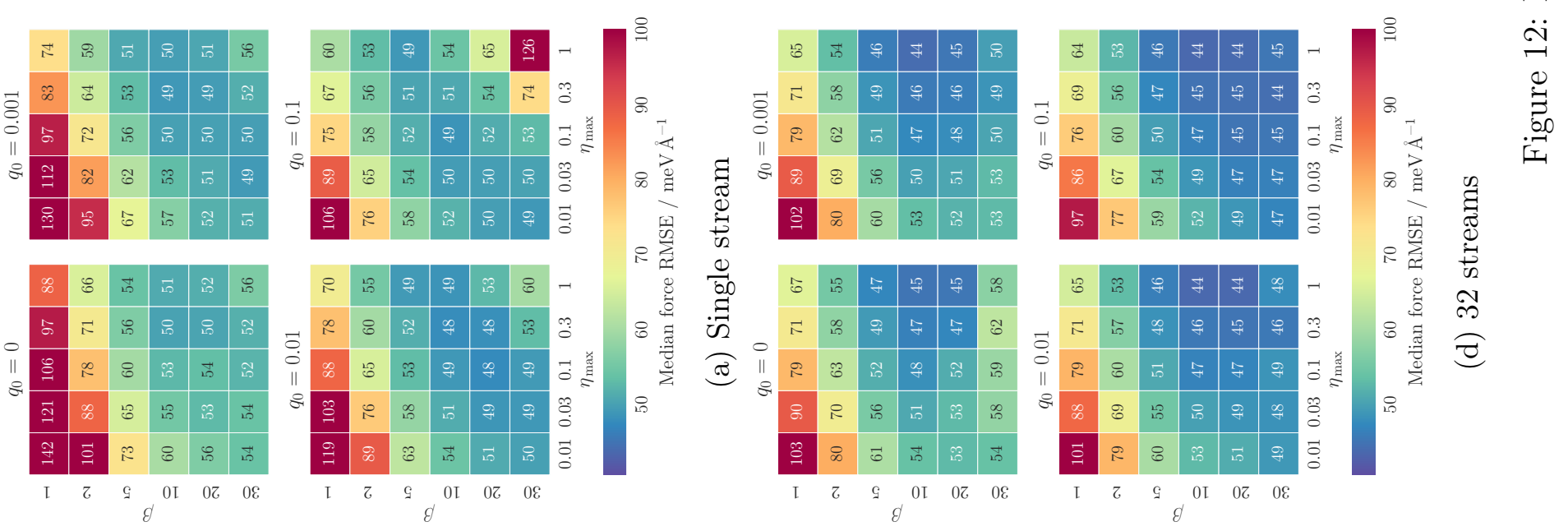


\section{Training time comparison}

In the main article Section 4.2 provides a comparison of the Kalman filter technique with first-order gradient-based methods. In analogy to Figure 5 in the publication we provide here learning curves for energy and force RMSEs but with the x-axis denoting the actual training time instead of the number of epochs. This view clearly shows the speedup if training is executed in parallel and mini-batches or multiple streams are used.

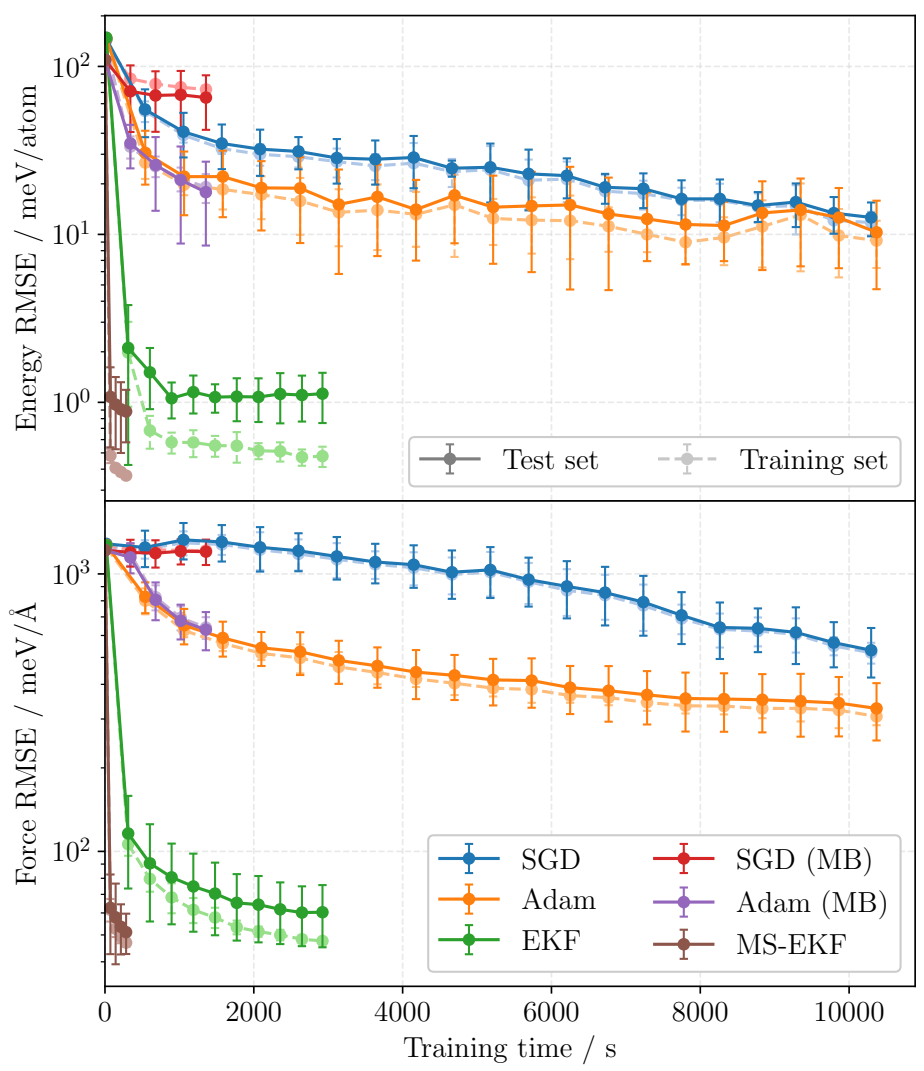

Figure 13: Performance comparison for different weight optimization algorithms. Training based on stochastic gradient descent (SGD) and Adam was executed for 1000 epochs, whereas the extended Kalman filter (EKF) training was stopped after only 100 epochs. The top and bottom panels show the learning curves in terms of energy and force RMSEs, respectively. Error bars represent the standard deviation calculated from multiple training runs with different random initialization. Results for the training sets are provided in light colors and dashed lines. A mini-batch size of 16 is indicated by "MB" for SGD and Adam in the legend. Similarly, the "MS-EKF" variant uses 16 streams for training. 


\section{$3 \quad \mathrm{Cu}_{2} \mathrm{~S}$ HDNNP symmetry function parameters}

Here we provide tables for the symmetry function parameter sets for the copper sulfide $\left(\mathrm{Cu}_{2} \mathrm{~S}\right)$ high-dimensional neural network potential described in the main text.

Table 1: Sulfur atom symmetry functions.

\begin{tabular}{|c|c|c|c|c|c|c|c|}
\hline$\#$ & $e_{1}$ & $e_{2}$ & $\eta / \AA^{-2}$ & $r_{s} / \AA$ & $\lambda$ & $\zeta$ & $r_{c} / \AA$ \\
\hline \multicolumn{8}{|c|}{$G^{\text {radial }}$} \\
\hline 1 & $\mathrm{~S}$ & - & 2.0 & 1.5 & - & - & 6.0 \\
\hline 2 & $\mathrm{Cu}$ & - & 2.0 & 1.5 & - & - & 6.0 \\
\hline 3 & $\mathrm{~S}$ & - & 2.0 & 2.0 & - & - & 6.0 \\
\hline 4 & $\mathrm{Cu}$ & - & 2.0 & 2.0 & - & - & 6.0 \\
\hline 5 & $\mathrm{~S}$ & - & 2.0 & 2.5 & - & - & 6.0 \\
\hline 6 & $\mathrm{Cu}$ & - & 2.0 & 2.5 & - & - & 6.0 \\
\hline 7 & $\mathrm{Cu}$ & - & 2.0 & 3.0 & - & - & 6.0 \\
\hline \multicolumn{8}{|c|}{$G^{\text {ang. n. }}$} \\
\hline 8 & $\mathrm{~S}$ & $\mathrm{~S}$ & 0.01653 & - & -1 & 1 & 6.0 \\
\hline 9 & $\mathrm{~S}$ & $\mathrm{Cu}$ & 0.01653 & - & -1 & 1 & 6.0 \\
\hline 10 & $\mathrm{Cu}$ & $\mathrm{Cu}$ & 0.01653 & - & -1 & 1 & 6.0 \\
\hline 11 & $\mathrm{~S}$ & $\mathrm{~S}$ & 0.01653 & - & 1 & 1 & 6.0 \\
\hline 12 & $\mathrm{~S}$ & $\mathrm{Cu}$ & 0.01653 & - & 1 & 1 & 6.0 \\
\hline 13 & $\mathrm{Cu}$ & $\mathrm{Cu}$ & 0.01653 & - & 1 & 1 & 6.0 \\
\hline 14 & $\mathrm{~S}$ & $\mathrm{~S}$ & 0.01653 & - & -1 & 6 & 6.0 \\
\hline 15 & $\mathrm{~S}$ & $\mathrm{Cu}$ & 0.01653 & - & -1 & 6 & 6.0 \\
\hline 16 & $\mathrm{Cu}$ & $\mathrm{Cu}$ & 0.01653 & - & -1 & 6 & 6.0 \\
\hline 17 & $\mathrm{~S}$ & $\mathrm{~S}$ & 0.01653 & - & 1 & 6 & 6.0 \\
\hline 18 & $\mathrm{~S}$ & $\mathrm{Cu}$ & 0.01653 & - & 1 & 6 & 6.0 \\
\hline 19 & $\mathrm{Cu}$ & $\mathrm{Cu}$ & 0.01653 & - & 1 & 6 & 6.0 \\
\hline 20 & $\mathrm{~S}$ & $\mathrm{~S}$ & 0.04082 & - & -1 & 1 & 6.0 \\
\hline 21 & $\mathrm{~S}$ & $\mathrm{Cu}$ & 0.04082 & - & -1 & 1 & 6.0 \\
\hline 22 & $\mathrm{Cu}$ & $\mathrm{Cu}$ & 0.04082 & - & -1 & 1 & 6.0 \\
\hline 23 & $\mathrm{~S}$ & $\mathrm{~S}$ & 0.04082 & - & 1 & 1 & 6.0 \\
\hline 24 & $\mathrm{~S}$ & $\mathrm{Cu}$ & 0.04082 & - & 1 & 1 & 6.0 \\
\hline 25 & $\mathrm{Cu}$ & $\mathrm{Cu}$ & 0.04082 & - & 1 & 1 & 6.0 \\
\hline 26 & $\mathrm{~S}$ & $\mathrm{~S}$ & 0.04082 & - & -1 & 6 & 6.0 \\
\hline 27 & $\mathrm{~S}$ & $\mathrm{Cu}$ & 0.04082 & - & -1 & 6 & 6.0 \\
\hline 28 & $\mathrm{Cu}$ & $\mathrm{Cu}$ & 0.04082 & - & -1 & 6 & 6.0 \\
\hline 29 & $\mathrm{~S}$ & $\mathrm{~S}$ & 0.04082 & - & 1 & 6 & 6.0 \\
\hline
\end{tabular}

Continued on next page 


\begin{tabular}{rrrccrcc}
$\#$ & $e_{1}$ & $e_{2}$ & $\eta / \AA^{-2}$ & $r_{s} / \AA$ & $\lambda$ & $\zeta$ & $r_{c} / \AA$ \\
\hline 30 & $\mathrm{~S}$ & $\mathrm{Cu}$ & 0.04082 & - & 1 & 6 & 6.0 \\
31 & $\mathrm{Cu}$ & $\mathrm{Cu}$ & 0.04082 & - & 1 & 6 & 6.0 \\
32 & $\mathrm{~S}$ & $\mathrm{~S}$ & 0.2222 & - & -1 & 1 & 6.0 \\
33 & $\mathrm{~S}$ & $\mathrm{Cu}$ & 0.2222 & - & -1 & 1 & 6.0 \\
34 & $\mathrm{~S}$ & $\mathrm{~S}$ & 0.2222 & - & 1 & 1 & 6.0 \\
35 & $\mathrm{~S}$ & $\mathrm{Cu}$ & 0.2222 & - & 1 & 1 & 6.0 \\
36 & $\mathrm{Cu}$ & $\mathrm{Cu}$ & 0.2222 & - & 1 & 1 & 6.0 \\
37 & $\mathrm{~S}$ & $\mathrm{~S}$ & 0.2222 & - & 1 & 6 & 6.0 \\
38 & $\mathrm{~S}$ & $\mathrm{Cu}$ & 0.2222 & - & 1 & 6 & 6.0 \\
\hline & & & $G$ ang. w. & & & \\
39 & $\mathrm{~S}$ & $\mathrm{~S}$ & 0.01653 & - & -1 & 1 & 6.0 \\
40 & $\mathrm{~S}$ & $\mathrm{Cu}$ & 0.01653 & - & -1 & 1 & 6.0 \\
41 & $\mathrm{Cu}$ & $\mathrm{Cu}$ & 0.01653 & - & -1 & 1 & 6.0 \\
42 & $\mathrm{~S}$ & $\mathrm{~S}$ & 0.01653 & - & 1 & 1 & 6.0 \\
43 & $\mathrm{~S}$ & $\mathrm{Cu}$ & 0.01653 & - & 1 & 1 & 6.0 \\
44 & $\mathrm{Cu}$ & $\mathrm{Cu}$ & 0.01653 & - & 1 & 1 & 6.0 \\
45 & $\mathrm{~S}$ & $\mathrm{~S}$ & 0.01653 & - & -1 & 6 & 6.0 \\
46 & $\mathrm{~S}$ & $\mathrm{Cu}$ & 0.01653 & - & -1 & 6 & 6.0 \\
47 & $\mathrm{Cu}$ & $\mathrm{Cu}$ & 0.01653 & - & -1 & 6 & 6.0 \\
48 & $\mathrm{~S}$ & $\mathrm{~S}$ & 0.01653 & - & 1 & 6 & 6.0 \\
49 & $\mathrm{~S}$ & $\mathrm{Cu}$ & 0.01653 & - & 1 & 6 & 6.0 \\
50 & $\mathrm{Cu}$ & $\mathrm{Cu}$ & 0.01653 & - & 1 & 6 & 6.0 \\
51 & $\mathrm{~S}$ & $\mathrm{~S}$ & 0.04082 & - & -1 & 1 & 6.0 \\
52 & $\mathrm{~S}$ & $\mathrm{Cu}$ & 0.04082 & - & -1 & 1 & 6.0 \\
53 & $\mathrm{Cu}$ & $\mathrm{Cu}$ & 0.04082 & - & -1 & 1 & 6.0 \\
54 & $\mathrm{~S}$ & $\mathrm{~S}$ & 0.04082 & - & 1 & 1 & 6.0 \\
55 & $\mathrm{~S}$ & $\mathrm{Cu}$ & 0.04082 & - & 1 & 1 & 6.0 \\
56 & $\mathrm{Cu}$ & $\mathrm{Cu}$ & 0.04082 & - & 1 & 1 & 6.0 \\
57 & $\mathrm{~S}$ & $\mathrm{~S}$ & 0.04082 & - & -1 & 6 & 6.0 \\
58 & $\mathrm{~S}$ & $\mathrm{Cu}$ & 0.04082 & - & -1 & 6 & 6.0 \\
59 & $\mathrm{Cu}$ & $\mathrm{Cu}$ & 0.04082 & - & -1 & 6 & 6.0 \\
60 & $\mathrm{~S}$ & $\mathrm{~S}$ & 0.04082 & - & 1 & 6 & 6.0 \\
61 & $\mathrm{~S}$ & $\mathrm{Cu}$ & 0.04082 & - & 1 & 6 & 6.0 \\
62 & $\mathrm{Cu}$ & $\mathrm{Cu}$ & 0.04082 & - & 1 & 6 & 6.0 \\
63 & $\mathrm{~S}$ & $\mathrm{~S}$ & 0.2222 & - & -1 & 1 & 6.0 \\
64 & $\mathrm{~S}$ & $\mathrm{Cu}$ & 0.2222 & - & -1 & 1 & 6.0 \\
65 & $\mathrm{Cu}$ & $\mathrm{Cu}$ & 0.2222 & - & -1 & 1 & 6.0 \\
66 & $\mathrm{~S}$ & $\mathrm{~S}$ & 0.2222 & - & 1 & 1 & 6.0 \\
\hline & & & $\mathrm{Continued}$ & $\mathrm{on}$ & next & $\mathrm{page}$ \\
\hline & & & & & & &
\end{tabular}




\begin{tabular}{rrrlcccc}
$\#$ & $e_{1}$ & $e_{2}$ & $\eta / \AA^{-2}$ & $r_{s} / \AA$ & $\lambda$ & $\zeta$ & $r_{c} / \AA$ \\
\hline 67 & $\mathrm{~S}$ & $\mathrm{Cu}$ & 0.2222 & - & 1 & 1 & 6.0 \\
68 & $\mathrm{Cu}$ & $\mathrm{Cu}$ & 0.2222 & - & 1 & 1 & 6.0 \\
69 & $\mathrm{~S}$ & $\mathrm{Cu}$ & 0.2222 & - & -1 & 6 & 6.0 \\
70 & $\mathrm{~S}$ & $\mathrm{~S}$ & 0.2222 & - & 1 & 6 & 6.0 \\
71 & $\mathrm{~S}$ & $\mathrm{Cu}$ & 0.2222 & - & 1 & 6 & 6.0 \\
72 & $\mathrm{Cu}$ & $\mathrm{Cu}$ & 0.2222 & - & 1 & 6 & 6.0 \\
\hline
\end{tabular}

Table 2: Copper atom symmetry functions.

\begin{tabular}{rrrccccc}
$\#$ & $e_{1}$ & $e_{2}$ & $\eta / \AA^{-2}$ & $r_{s} / \AA$ & $\lambda$ & $\zeta$ & $r_{c} / \AA$ \\
\hline \hline & & \multicolumn{7}{c}{$G^{\text {radial }}$} & & & \\
1 & $\mathrm{~S}$ & - & 2.0 & 1.5 & - & - & 6.0 \\
2 & $\mathrm{~S}$ & - & 2.0 & 2.0 & - & - & 6.0 \\
3 & $\mathrm{Cu}$ & - & 2.0 & 2.0 & - & - & 6.0 \\
4 & $\mathrm{~S}$ & - & 2.0 & 2.5 & - & - & 6.0 \\
5 & $\mathrm{Cu}$ & - & 2.0 & 2.5 & - & - & 6.0 \\
6 & $\mathrm{~S}$ & - & 2.0 & 3.0 & - & - & 6.0 \\
7 & $\mathrm{Cu}$ & - & 2.0 & 3.0 & - & - & 6.0 \\
8 & $\mathrm{~S}$ & - & 2.0 & 4.0 & - & - & 6.0 \\
9 & $\mathrm{~S}$ & - & 2.0 & 5.0 & - & - & 6.0 \\
\hline & & & $G$ & ang. n. & & & \\
10 & $\mathrm{~S}$ & $\mathrm{~S}$ & 0.01653 & - & -1 & 1 & 6.0 \\
11 & $\mathrm{~S}$ & $\mathrm{Cu}$ & 0.01653 & - & -1 & 1 & 6.0 \\
12 & $\mathrm{Cu}$ & $\mathrm{Cu}$ & 0.01653 & - & -1 & 1 & 6.0 \\
13 & $\mathrm{~S}$ & $\mathrm{~S}$ & 0.01653 & - & 1 & 1 & 6.0 \\
14 & $\mathrm{~S}$ & $\mathrm{Cu}$ & 0.01653 & - & 1 & 1 & 6.0 \\
15 & $\mathrm{Cu}$ & $\mathrm{Cu}$ & 0.01653 & - & 1 & 1 & 6.0 \\
16 & $\mathrm{~S}$ & $\mathrm{~S}$ & 0.01653 & - & -1 & 6 & 6.0 \\
17 & $\mathrm{Cu}$ & $\mathrm{Cu}$ & 0.01653 & - & -1 & 6 & 6.0 \\
18 & $\mathrm{~S}$ & $\mathrm{~S}$ & 0.01653 & - & 1 & 6 & 6.0 \\
19 & $\mathrm{~S}$ & $\mathrm{Cu}$ & 0.01653 & - & 1 & 6 & 6.0 \\
20 & $\mathrm{Cu}$ & $\mathrm{Cu}$ & 0.01653 & - & 1 & 6 & 6.0 \\
21 & $\mathrm{~S}$ & $\mathrm{~S}$ & 0.04082 & - & -1 & 1 & 6.0 \\
22 & $\mathrm{~S}$ & $\mathrm{Cu}$ & 0.04082 & - & -1 & 1 & 6.0 \\
23 & $\mathrm{Cu}$ & $\mathrm{Cu}$ & 0.04082 & - & -1 & 1 & 6.0 \\
24 & $\mathrm{~S}$ & $\mathrm{~S}$ & 0.04082 & - & 1 & 1 & 6.0 \\
\hline
\end{tabular}

Continued on next page 


\begin{tabular}{|c|c|c|c|c|c|c|c|}
\hline$\#$ & $e_{1}$ & $e_{2}$ & $\eta / \AA^{-2}$ & $r_{s} / \AA$ & $\lambda$ & & $r_{c} / \AA$ \\
\hline 25 & $\mathrm{~S}$ & $\mathrm{Cu}$ & 0.04082 & - & 1 & 1 & 6.0 \\
\hline 26 & $\mathrm{Cu}$ & $\mathrm{Cu}$ & 0.04082 & - & 1 & 1 & 6.0 \\
\hline 27 & $\mathrm{~S}$ & S & 0.04082 & - & -1 & 6 & 6.0 \\
\hline 28 & $\mathrm{Cu}$ & $\mathrm{Cu}$ & 0.04082 & - & -1 & 6 & 6.0 \\
\hline 29 & $\mathrm{~S}$ & S & 0.04082 & - & 1 & 6 & 6.0 \\
\hline 30 & S & $\mathrm{Cu}$ & 0.04082 & - & 1 & 6 & 6.0 \\
\hline 31 & $\mathrm{Cu}$ & $\mathrm{Cu}$ & 0.04082 & - & 1 & 6 & 6.0 \\
\hline 32 & $\mathrm{~S}$ & $\mathrm{~S}$ & 0.2222 & - & -1 & 1 & 6.0 \\
\hline 33 & $\mathrm{~S}$ & S & 0.2222 & - & 1 & 1 & 6.0 \\
\hline 34 & S & S & 0.2222 & - & 1 & 6 & 6.0 \\
\hline \multicolumn{8}{|c|}{$G^{\text {ang.w. }}$} \\
\hline 35 & S & S & 0.01653 & - & -1 & 1 & 6.0 \\
\hline 36 & $\mathrm{~S}$ & $\mathrm{Cu}$ & 0.01653 & - & -1 & 1 & 6.0 \\
\hline 37 & $\mathrm{Cu}$ & $\mathrm{Cu}$ & 0.01653 & 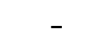 & -1 & 1 & 6.0 \\
\hline 38 & S & S & 0.01653 & - & 1 & 1 & 6.0 \\
\hline 39 & $\mathrm{~S}$ & $\mathrm{Cu}$ & 0.01653 & - & 1 & 1 & 6.0 \\
\hline 40 & $\mathrm{Cu}$ & $\mathrm{Cu}$ & 0.01653 & - & 1 & 1 & 6.0 \\
\hline 41 & S & S & 0.01653 & - & -1 & 6 & 6.0 \\
\hline 42 & S & $\mathrm{Cu}$ & 0.01653 & - & -1 & 6 & 6.0 \\
\hline 43 & $\mathrm{Cu}$ & $\mathrm{Cu}$ & 0.01653 & - & -1 & 6 & 6.0 \\
\hline 44 & $\mathrm{~S}$ & S & 0.01653 & - & 1 & 6 & 6.0 \\
\hline 45 & $\mathrm{~S}$ & $\mathrm{Cu}$ & 0.01653 & - & 1 & 6 & 6.0 \\
\hline 46 & $\mathrm{Cu}$ & $\mathrm{Cu}$ & 0.01653 & - & 1 & 6 & 6.0 \\
\hline 47 & S & S & 0.04082 & - & -1 & 1 & 6.0 \\
\hline 48 & S & $\mathrm{Cu}$ & 0.04082 & - & -1 & 1 & 6.0 \\
\hline 49 & $\mathrm{Cu}$ & $\mathrm{Cu}$ & 0.04082 & - & -1 & 1 & 6.0 \\
\hline 50 & S & S & 0.04082 & - & 1 & 1 & 6.0 \\
\hline 51 & S & $\mathrm{Cu}$ & 0.04082 & - & 1 & 1 & 6.0 \\
\hline 52 & $\mathrm{Cu}$ & $\mathrm{Cu}$ & 0.04082 & - & 1 & 1 & 6.0 \\
\hline 53 & S & S & 0.04082 & - & -1 & 6 & 6.0 \\
\hline 54 & S & $\mathrm{Cu}$ & 0.04082 & - & -1 & 6 & 6.0 \\
\hline 55 & $\mathrm{Cu}$ & $\mathrm{Cu}$ & 0.04082 & - & -1 & 6 & 6.0 \\
\hline 56 & S & $\mathrm{S}$ & 0.04082 & - & 1 & 6 & 6.0 \\
\hline 57 & S & $\mathrm{Cu}$ & 0.04082 & - & 1 & 6 & 6.0 \\
\hline 58 & $\mathrm{Cu}$ & $\mathrm{Cu}$ & 0.04082 & - & 1 & 6 & 6.0 \\
\hline 59 & S & S & 0.2222 & - & -1 & 1 & 6.0 \\
\hline 60 & S & $\mathrm{Cu}$ & 0.2222 & - & -1 & 1 & 6.0 \\
\hline 61 & $\mathrm{Cu}$ & $\mathrm{Cu}$ & 0.2222 & - & -1 & 1 & 6.0 \\
\hline
\end{tabular}




\begin{tabular}{rrrccccc}
$\#$ & $e_{1}$ & $e_{2}$ & $\eta / \AA^{-2}$ & $r_{s} / \AA$ & $\lambda$ & $\zeta$ & $r_{c} / \AA$ \\
\hline 62 & $\mathrm{~S}$ & $\mathrm{~S}$ & 0.2222 & - & 1 & 1 & 6.0 \\
63 & $\mathrm{~S}$ & $\mathrm{Cu}$ & 0.2222 & - & 1 & 1 & 6.0 \\
64 & $\mathrm{Cu}$ & $\mathrm{Cu}$ & 0.2222 & - & 1 & 1 & 6.0 \\
65 & $\mathrm{~S}$ & $\mathrm{~S}$ & 0.2222 & - & 1 & 6 & 6.0 \\
66 & $\mathrm{~S}$ & $\mathrm{Cu}$ & 0.2222 & - & 1 & 6 & 6.0 \\
\hline
\end{tabular}

\section{Low chalcocite structure comparison}

The COMPSTRU ${ }^{1 / 2}$ application part of the Bilbao Crystallographic Server ${ }^{\sqrt[3]{5}}$ returns all crystallographic information about symmetries and occupied Wyckoff sites in the CIF format. For reference, we provide the output for the low chalcocite configuration determined 1979 by Evans ${ }^{6}$ (low-chalcocite_Evans-1979.cif) and the closely matching HDNNP equilibrium structure (low-chalcocite_HDNNP.cif). Table 3 shows the deviations between these two configurations as computed by COMPSTRU. 
Table 3: Deviations of sulfur and copper atoms in each direction (fractional coordinates $u_{x}, u_{y}, u_{z}$ ) and the absolute distance in $\AA$.

\begin{tabular}{|c|c|c|c|c|c|}
\hline Atom & WP & $u_{x}$ & $u_{y}$ & $u_{z}$ & $d / \AA$ \\
\hline S1 & 4e $(x, y, z)$ & 0.0019 & 0.0005 & 0.0002 & 0.0283 \\
\hline $\mathrm{S} 2$ & $4 \mathrm{e}(\mathrm{x}, \mathrm{y}, \mathrm{z})$ & -0.0019 & 0.0022 & -0.0041 & 0.0563 \\
\hline S3 & $4 \mathrm{e}(\mathrm{x}, \mathrm{y}, \mathrm{z})$ & -0.0059 & 0.0020 & -0.0003 & 0.0909 \\
\hline S4 & $4 \mathrm{e}(\mathrm{x}, \mathrm{y}, \mathrm{z})$ & -0.0024 & 0.0064 & 0.0028 & 0.0988 \\
\hline S5 & $4 \mathrm{e}(\mathrm{x}, \mathrm{y}, \mathrm{z})$ & -0.0004 & 0.0004 & -0.0027 & 0.0340 \\
\hline S6 & 4e $(x, y, z)$ & 0.0018 & 0.0043 & 0.0043 & 0.0726 \\
\hline S7 & $4 \mathrm{e}(\mathrm{x}, \mathrm{y}, \mathrm{z})$ & 0.0015 & 0.0047 & -0.0026 & 0.0748 \\
\hline S8 & $4 \mathrm{e}(\mathrm{x}, \mathrm{y}, \mathrm{z})$ & -0.0076 & 0.0016 & -0.0068 & 0.1133 \\
\hline S9 & $4 \mathrm{e}(\mathrm{x}, \mathrm{y}, \mathrm{z})$ & -0.0011 & 0.0032 & -0.0041 & 0.0631 \\
\hline S10 & $4 \mathrm{e}(\mathrm{x}, \mathrm{y}, \mathrm{z})$ & -0.0008 & 0.0058 & -0.0019 & 0.0723 \\
\hline S11 & $4 \mathrm{e}(\mathrm{x}, \mathrm{y}, \mathrm{z})$ & -0.0006 & 0.0036 & 0.0006 & 0.0448 \\
\hline S12 & $4 \mathrm{e}(\mathrm{x}, \mathrm{y}, \mathrm{z})$ & -0.0017 & 0.0029 & 0.0005 & 0.0454 \\
\hline $\mathrm{Cu} 1$ & $4 \mathrm{e}(\mathrm{x}, \mathrm{y}, \mathrm{z})$ & -0.0011 & 0.0008 & 0.0005 & 0.0225 \\
\hline $\mathrm{Cu} 2$ & $4 \mathrm{e}(\mathrm{x}, \mathrm{y}, \mathrm{z})$ & -0.0012 & 0.0044 & -0.0021 & 0.0589 \\
\hline $\mathrm{Cu} 3$ & $4 \mathrm{e}(\mathrm{x}, \mathrm{y}, \mathrm{z})$ & -0.0004 & -0.0042 & 0.0037 & 0.0735 \\
\hline $\mathrm{Cu} 4$ & $4 \mathrm{e}(\mathrm{x}, \mathrm{y}, \mathrm{z})$ & 0.0011 & 0.0030 & -0.0007 & 0.0420 \\
\hline $\mathrm{Cu} 5$ & 4e $(x, y, z)$ & 0.0017 & -0.0051 & 0.0068 & 0.1028 \\
\hline $\mathrm{Cu} 6$ & $4 \mathrm{e}(\mathrm{x}, \mathrm{y}, \mathrm{z})$ & -0.0023 & 0.0046 & -0.0015 & 0.0633 \\
\hline $\mathrm{Cu} 7$ & $4 \mathrm{e}(\mathrm{x}, \mathrm{y}, \mathrm{z})$ & -0.0051 & -0.0022 & -0.0013 & 0.0765 \\
\hline $\mathrm{Cu} 8$ & $4 \mathrm{e}(\mathrm{x}, \mathrm{y}, \mathrm{z})$ & -0.0033 & -0.0038 & -0.0034 & 0.0682 \\
\hline $\mathrm{Cu} 9$ & $4 \mathrm{e}(\mathrm{x}, \mathrm{y}, \mathrm{z})$ & 0.0030 & -0.0024 & -0.0003 & 0.0560 \\
\hline $\mathrm{Cu} 10$ & $4 \mathrm{e}(\mathrm{x}, \mathrm{y}, \mathrm{z})$ & -0.0001 & -0.0065 & -0.0003 & 0.0770 \\
\hline Cu11 & $4 \mathrm{e}(\mathrm{x}, \mathrm{y}, \mathrm{z})$ & -0.0055 & -0.0007 & -0.0055 & 0.0839 \\
\hline $\mathrm{Cu} 12$ & $4 \mathrm{e}(\mathrm{x}, \mathrm{y}, \mathrm{z})$ & 0.0029 & 0.0011 & 0.0006 & 0.0430 \\
\hline $\mathrm{Cu} 13$ & $4 \mathrm{e}(\mathrm{x}, \mathrm{y}, \mathrm{z})$ & 0.0062 & -0.0051 & 0.0075 & 0.1205 \\
\hline $\mathrm{Cu} 14$ & $4 \mathrm{e}(\mathrm{x}, \mathrm{y}, \mathrm{z})$ & 0.0023 & -0.0040 & 0.0056 & 0.0826 \\
\hline $\mathrm{Cu} 15$ & $4 \mathrm{e}(\mathrm{x}, \mathrm{y}, \mathrm{z})$ & -0.0057 & 0.0025 & 0.0012 & 0.0992 \\
\hline $\mathrm{Cu} 16$ & $4 \mathrm{e}(\mathrm{x}, \mathrm{y}, \mathrm{z})$ & -0.0026 & -0.0026 & 0.0006 & 0.0534 \\
\hline $\mathrm{Cu} 17$ & $4 \mathrm{e}(\mathrm{x}, \mathrm{y}, \mathrm{z})$ & -0.0016 & -0.0122 & -0.0046 & 0.1552 \\
\hline $\mathrm{Cu} 18$ & $4 \mathrm{e}(\mathrm{x}, \mathrm{y}, \mathrm{z})$ & 0.0057 & -0.0050 & -0.0004 & 0.1065 \\
\hline Cu19 & $4 \mathrm{e}(\mathrm{x}, \mathrm{y}, \mathrm{z})$ & 0.0011 & 0.0006 & -0.0015 & 0.0328 \\
\hline $\mathrm{Cu} 20$ & $4 \mathrm{e}(\mathrm{x}, \mathrm{y}, \mathrm{z})$ & -0.0038 & -0.0033 & -0.0046 & 0.0749 \\
\hline $\mathrm{Cu} 21$ & $4 \mathrm{e}(\mathrm{x}, \mathrm{y}, \mathrm{z})$ & -0.0010 & -0.0053 & -0.0021 & 0.0684 \\
\hline $\mathrm{Cu} 22$ & 4e $(\mathrm{x}, \mathrm{y}, \mathrm{z})$ & 0.0023 & -0.0043 & -0.0015 & 0.0697 \\
\hline $\mathrm{Cu} 23$ & 4e $(\mathrm{x}, \mathrm{y}, \mathrm{z})$ & 0.0032 & -0.0057 & 0.0022 & 0.0817 \\
\hline $\mathrm{Cu} 24$ & $4 \mathrm{e}(\mathrm{x}, \mathrm{y}, \mathrm{z})$ & 0.0005 & -0.0033 & -0.0023 & 0.0522 \\
\hline
\end{tabular}




\section{Copper diffusion coefficient values}

Table 4 lists copper diffusion coefficients corresponding to Figure 8 in the main text.

Table 4: Copper diffusion coefficients depending on temperature. The column "phase" labels whether the system is in the low- $(\alpha \mathrm{Ch})$ or high- $(\beta \mathrm{Ch})$ chalcocite phase. The MD trajectory length is given in the column $t_{\text {Traj. }}$.

\begin{tabular}{cccc}
\hline$T / \mathrm{K}$ & phase & $D_{\mathrm{Cu}} / \mathrm{cm}^{2} / \mathrm{s}$ & $t_{\text {Traj. }} / \mathrm{ns}$ \\
\hline 330 & $\alpha \mathrm{Ch}$ & $1.77 \times 10^{-9}$ & 10.0 \\
340 & $\alpha \mathrm{Ch}$ & $4.03 \times 10^{-9}$ & 10.0 \\
350 & $\alpha \mathrm{Ch}$ & $7.77 \times 10^{-9}$ & 10.0 \\
360 & $\alpha \mathrm{Ch}$ & $1.28 \times 10^{-8}$ & 10.0 \\
370 & $\alpha \mathrm{Ch}$ & $2.57 \times 10^{-8}$ & 10.0 \\
375 & $\alpha \mathrm{Ch}$ & $3.54 \times 10^{-8}$ & 10.0 \\
380 & $\alpha \mathrm{Ch}$ & $5.44 \times 10^{-8}$ & 10.0 \\
350 & $\beta \mathrm{Ch}$ & $5.57 \times 10^{-7}$ & 0.5 \\
375 & $\beta \mathrm{Ch}$ & $8.98 \times 10^{-7}$ & 0.5 \\
385 & $\beta \mathrm{Ch}$ & $1.20 \times 10^{-6}$ & 0.5 \\
390 & $\beta \mathrm{Ch}$ & $1.15 \times 10^{-6}$ & 0.5 \\
395 & $\beta \mathrm{Ch}$ & $1.31 \times 10^{-6}$ & 0.5 \\
400 & $\beta \mathrm{Ch}$ & $1.52 \times 10^{-6}$ & 0.5 \\
405 & $\beta \mathrm{Ch}$ & $1.57 \times 10^{-6}$ & 0.5 \\
410 & $\beta \mathrm{Ch}$ & $1.73 \times 10^{-6}$ & 0.5 \\
425 & $\beta \mathrm{Ch}$ & $2.19 \times 10^{-6}$ & 0.5 \\
450 & $\beta \mathrm{Ch}$ & $2.97 \times 10^{-6}$ & 0.5 \\
475 & $\beta \mathrm{Ch}$ & $3.50 \times 10^{-6}$ & 0.5 \\
500 & $\beta \mathrm{Ch}$ & $4.63 \times 10^{-6}$ & 0.5 \\
525 & $\beta \mathrm{Ch}$ & $5.85 \times 10^{-6}$ & 0.5 \\
550 & $\beta \mathrm{Ch}$ & $7.32 \times 10^{-6}$ & 5.0 \\
\hline
\end{tabular}




\section{References}

${ }^{1}$ E. S. Tasci, G. de la Flor, D. Orobengoa, C. Capillas, J. M. Perez-Mato, and M. I. Aroyo, "An introduction to the tools hosted in the Bilbao Crystallographic Server," EPJ Web of Conferences, vol. 22, p. 00009, 2012.

${ }^{2}$ G. de la Flor, D. Orobengoa, E. Tasci, J. M. Perez-Mato, and M. I. Aroyo, "Comparison of structures applying the tools available at the Bilbao Crystallographic Server," Journal of Applied Crystallography, vol. 49, pp. 653-664, Apr. 2016.

${ }^{3}$ M. I. Aroyo, A. Kirov, C. Capillas, J. M. Perez-Mato, and H. Wondratschek, "Bilbao Crystallographic Server. II. Representations of crystallographic point groups and space groups," Acta Crystallographica Section A: Foundations of Crystallography, vol. 62, pp. 115-128, Mar. 2006.

${ }^{4}$ M. I. Aroyo, J. Perez-Mato, D. Orobengoa, E. Tasci, G. De La Flor, and A. Kirov, "Crystallography online: Bilbao crystallographic server," Bulgarian Chemical Communications, vol. 43, no. 2, pp. 183-197, 2011.

${ }^{5}$ M. I. Aroyo, J. M. Perez-Mato, C. Capillas, E. Kroumova, S. Ivantchev, G. Madariaga, A. Kirov, and H. Wondratschek, "Bilbao Crystallographic Server: I. Databases and crystallographic computing programs," Zeitschrift für Kristallographie - Crystalline Materials, vol. 221, no. 1, pp. 15-27, 2009.

${ }^{6}$ H. T. Evans, "The crystal structures of low chalcocite and djurleite," Zeitschrift für Kristallographie-Crystalline Materials, vol. 150, no. 1-4, pp. 299-320, 1979. 